\title{
Optimal Fault Tolerant Control of Large-Scale Wind Turbines in the Case of the Pitch Actuator Partial Faults
}

\author{
Younes Ait El Maati (iD) and Lhoussain El Bahir ii \\ Cadi Ayad University, Laboratory of Electrical Engineering and Control Systems (LGECOS), National School of Applied Sciences, \\ Av. Abdelkarim El Khattabi B.P 575, Marrakech 40000, Morocco
}

Correspondence should be addressed to Younes Ait El Maati; aitelmaati.younes@gmail.com

Received 24 September 2019; Revised 30 December 2019; Accepted 11 January 2020; Published 12 February 2020

Guest Editor: Kiyong Oh

Copyright (c) 2020 Younes Ait El Maati and Lhoussain El Bahir. This is an open access article distributed under the Creative Commons Attribution License, which permits unrestricted use, distribution, and reproduction in any medium, provided the original work is properly cited.

\begin{abstract}
In this paper, an adaptive fault tolerant control strategy is proposed to deal with the three pitch actuator faults in the largescale wind turbines. Firstly, a simultaneous state and fault estimation was performed through a suitable LMI (linear matrix inequality) based optimal strategy. Hereafter, the new control law is designed using the previously estimated fault information. The actuator efficiency estimator uses as design parameters, respectively, the performance index $\gamma$ against the wind and the learning rate $\Xi$. of the fault estimation algorithm. The study shows that the choice of the previous two parameters impacts the response time of the fault estimation and the correlation of the tracking error with the wind. The aim is to choose a small fault estimation response time while keeping a weak correlation between the tracking error and the wind turbulence noise. Finally, a tuning strategy is elaborated to choose the suitable $\gamma$ and $\Xi$ to match the reconfiguration objective.
\end{abstract}

\section{Introduction}

The increasing energy demand leads to the production of large wind turbines. Thus, more and more vibrational behaviors could be expected in the different degrees of freedom (DOFs). Figures 1 and 2 show the most important DOF. The excitation of the DOFs leads to increase in loads and forces on the structure. The aim is to reduce the loads in order to protect the structure against fatigue and damage.

The wind turbine operates in two different regions, low and high winds. In the first region, so called partial load region, the aim is to maximize the extracted power $[1,2]$. This was achieved by controlling the generator torque to maintain an optimum ratio between the tip speed of the blades and the operating wind speed [3]. In the second region, so called full load region, the wind turbine was controlled to reduce loads and keep the power at its rated value. Practically, the constant power was maintained through a fixed speed and a fixed generator torque. This has been obtained by pitching the blades from or toward wind to adjust the speed of the rotor, while applying a constant generator torque [4]. For example, the authors in [5] proposed an anticipative control scheme of a wind turbine working in the maximum power region. The idea is to use the wind speed as a generator of the set point of the MPP (maximal power point) control.

1.1. The Pitch Actuator Control of Wind Turbines in the Full Load Region. In the full load region, the high turbulence occurs and produces big deflection movements on the blades. The pitch actuators were used to attenuate the loads in the full load region [6-8].

Many papers have studied the loads exerted by the wind on the different parts of the wind turbine structure. In [9], the authors discussed and identified which wind turbine components can benefit from advanced control algorithms and also presented results from a preliminary loads case analysis using a baseline controller. 


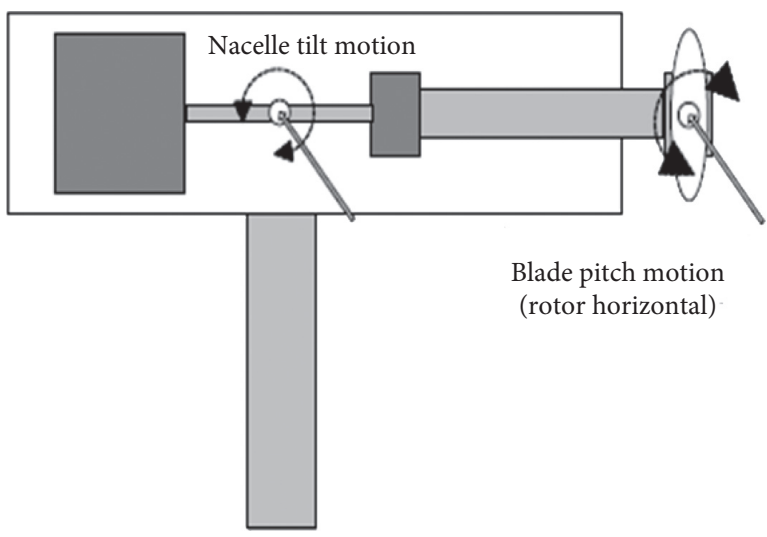

Figure 1: Blade pitch degree of freedom.

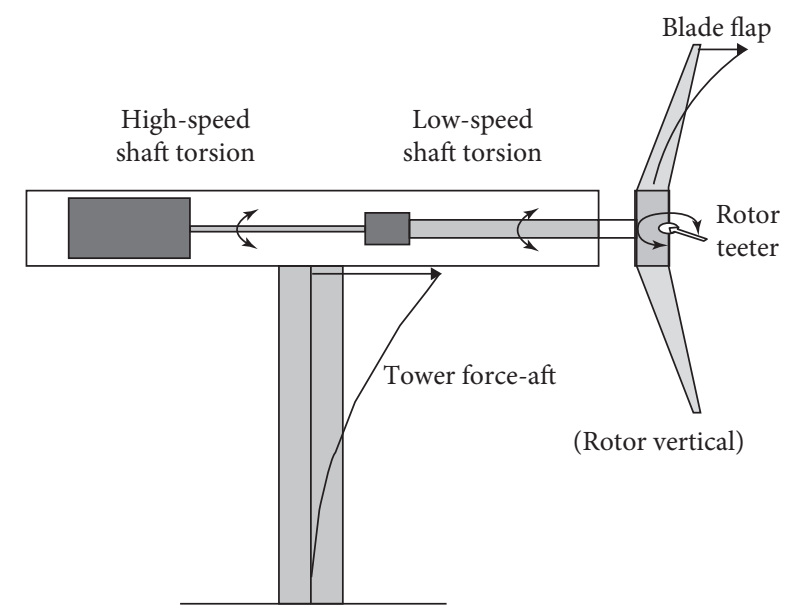

Figure 2: Blade flap and tower fore-aft degrees of freedom.

From the discussed papers above, it can be noticed that the wind turbines in the megawatt size are expensive, and hence their reliability is expected to be high to generate as much energy as possible. This type of wind turbines is expected to have reduced downtimes while keeping energy production. Usually, the turbines are turned off even during simple faults to wait for service.

\subsection{Fault Detection and Tolerant Control in Wind Turbines.} The statistics in [10] show that gearbox faults are the most critical ones in wind turbines, essentially due to its relatively high stopping time and severity on the whole structure. Another type of wind turbine faults is of electrical root. For example, the wind turbine can face short circuit faults in the wires or inverter over voltage faults. It can also experience hard over fault in the generator or some sensor faults like drift or bias.

The last reason creates the need to introduce fault detection, isolation, and accommodation systems within the existing classical control systems in order to reduce stop time even with limited energy production [11]. Early detection has been the spot of the control industry studies especially using residual generation and fault analysis. The paper [12] presents an overview of the fault diagnosis method categories, with an application to the wind turbine pitch fault control. For more details, see also [13, 14].

The model-based methods are one of the most suitable methods for the study of the fault tolerant control of the wind turbines. In fact, the availability of the large-scale wind turbine model is no longer a problem especially with the existence of highly trusted wind environment software and emulators such as FAST (Fatigue, Aerodynamics, Structures, and Turbulence).

In this paper, the FAST code is used to test the proposed strategy. FAST is an aeroelastic simulator capable of predicting the fatigue loads of two- and three-bladed flexible with 24 degrees of freedom $[15,16]$. The code uses a modal approach in combination with Kane dynamics to develop the equations of motion. FAST helps considerably researchers and engineers in testing wind load reduction algorithms [17]. This software provides a linearized model parameter about a chosen operating point with more than 40 measures of outputs (rotor speed, generator speed, and tower and blade displacement).

The pitch servomotor is the most critical actuator in the wind turbine [18, 19]. As discussed earlier, it is used to regulate the power and to attenuate the vibrations in the high wind region. If this actuator loses its efficiency, it will no longer provide the sufficient control efforts to attenuate the vibrations and regulate the power. Based on the last reason, a new control law is to be constructed to deal with the fault. The new control efforts could be produced by online tuning of the controller parameters or by changing the baseline control law using an estimation of the fault [20-22].

In the wind turbines' control field, in the pitch actuator fault, the authors inreference [23] proposed a fault tolerant control strategy to deal with the drop of the hydraulic liquid in the servo pitches of the rotor. In the same field, another type of fault is the imbalance in the rotor due to icing or destruction of one or more blades. This fault not only reduces the aerodynamic efficiency of the turbine and its power output but can also lead to large increases of loads on the drivetrain, blades, and tower. For this, the authors in [24] used the FAST software to model aerodynamic imbalance in a sample $5 \mathrm{MW}$ offshore wind turbine. It has been concluded that the combination of blade and nacelle measurements, obtained from instrumentation already placed on the structure, can be formulated into an algorithm used to detect and locate the imbalance (see also [25]).

Another essential component is the anemometer. The anemometers are used in the feedforward control of the wind turbines. In [26], the authors proposed a new method to estimate the wind speed that could be substituted for anemometers in control loops. Moreover, the authors in [27] proposed a robust observer to generate residuals for the fault turbine moment sensors which provides a measurement of the loads applied on the structure. In [28], the authors have proposed a method to estimate the gearbox efficiency in the wind turbines. The idea is to detect the drop in the efficiency and generate a suitable residual to indicate the fault occurrence (see also $[29,30]$ ).

Given the very disturbed wind nature, several optimization methods could be used to minimize the effect of the 
disturbance on the outputs. In [31], a multiagent optimal method based on reinforcement learning was proposed to control a grid connected energy system.

The fault isolation and tolerant control applied to the wind turbines has been illustrated in many papers. For example, in [32,33], a codesign of the observers and decision mechanisms for satisfying certain trade-off between different isolation performance indices has been established. The performance indices were the false isolation rates, the isolation times, and the minimum size of the isolable faults. The considered fault was additive on the pitch actuator. The paper [34] used jump observers to detect and isolate the faults. The false alarm rate is upper bounded by means of Markov's inequality. The trade-offs between the minimum detectable faults, the false alarm rate, and the response time to faults of the fault diagnoser have been explored. In the previous cited works, the assumption of slow dynamics faults was considered which limits the usage of the strategies to slow faults dynamics.

In this paper, the following contributions have been developed:

(i) An integrator-based simultaneous states and fault estimator algorithm is designed.

(ii) The stability and the robustness of the algorithm is proven for the partial fault of the pitch actuators using Lyapunov theory.

(iii) A fault tolerant control including a baseline multiobjective state feedback regulator is designed. The performance of the method depends on two parameters to be tuned, $\gamma$ and $\Xi$.

(iv) To ensure a best performance of the proposed control solution, two mappings are proposed to tune the design parameters $\gamma$ and $\Xi$.

(v) The evaluation of the proposed control strategy is performed on the $1.5 \mathrm{MW}$ wind turbine benchmark model.

This paper is organized as follows. In Section 2, the wind turbine model used in the benchmark is presented. In Section 3, the wind turbine fault and state estimation is performed and the nominal multiobjective regulator is designed. The wind turbine adaptive fault tolerant control in the faulty production is studied and the tuning strategy is proposed in Section 4. Some concluding remarks are presented in Section 5.

\section{The Wind Turbine Model}

The wind turbine model used for the control contains seven wind turbine states as follows:

(i) The rotor speed

(ii) The blade $i$ flap wise deflection

(iii) The blade $i$ flap wise deflection velocity

where $i=1,2,3$.

The state space model of the rotor about the operating point is given by

$$
\left\{\begin{array}{c}
\delta \dot{x}=A \delta x+B \delta u+D_{d} \omega \\
\delta y=C \delta x
\end{array}\right\} .
$$

The operator $\delta$ means a variation about the operating point. $A$ is the dynamics matrix, $B$ is the input matrix, $D_{d}$ is the disturbance matrix, and $C$ is the measured output matrix. The numerical values of the matrices $A, B, C$, and $D_{d}$ are given in the Appendix.

The state vector and the pitch actions are, respectively, represented by the vectors $\delta x$ and $\delta u$ :

$$
\begin{aligned}
\delta x & =\left[\begin{array}{lll}
\delta \Omega_{r} \delta x_{-} \operatorname{def}_{1} \delta \dot{x}_{-} \operatorname{def}_{1} \delta x_{-} \operatorname{def}_{2} \delta \dot{x}_{-} \operatorname{def}_{2} \delta x_{-} \operatorname{def}_{3} \delta \dot{x}_{-} \operatorname{def}_{3}
\end{array}\right]^{T}, \\
\delta u & =\left[\begin{array}{lll}
\delta \beta_{1} & \delta \beta_{2} & \delta \beta_{3}
\end{array}\right]^{T},
\end{aligned}
$$

where $\delta x_{-} \operatorname{def}_{i}$ and $\delta \dot{x}_{-} \operatorname{def}_{i} \dot{x}_{-}$def $_{i}$ are, respectively, the blade deflection and deflection velocity associated to the $i^{\text {th }}$ blade. $\delta y$ represents the measured rotor speed and deflection velocity. The vector $\omega$ is the wind disturbance on the considered states and is given by

$$
\omega=V(t)-V_{0},
$$

where $V(t)$ is the instantaneous wind speed and $V_{0}$ is the operating wind speed which is equal to $18 \mathrm{~m} / \mathrm{s}$ in the high wind region. The vector is the rotor speed about the operating point and is given by

$$
\delta \Omega_{r}=\Omega_{r}(t)-\Omega_{r 0},
$$

where $\Omega_{r}(t)$ is the measured rotor speed and the operating rotor speed is $\Omega_{r 0}=40 \mathrm{rpm}$. The input pitches about operating point are equal to $\delta \beta_{1}=\delta \beta_{2}=\delta \beta_{3}=\beta(t)-\beta_{0}$. $\beta(t)$ is the measured pitch angle and the operating pitch angle is $\beta_{0}=9^{\circ}$.

The numerical values of variables in the international system of measurement units are given.

It is assumed that the measured outputs are the rotor speed and the blade flap deflection velocities of the three blades. In actual application like wind turbine blade analysis, the deflection velocities could be obtained using the derivative of the deflection displacement measure. This information could be directly obtained using a MEMS gyroscope sensor as in [35], or estimated using ultrawideband signals as proposed in [36].

The considered turbine is a Wind PACT 1.5 MW large scale. More details about the parameters of this wind turbine could be found in $[37,38]$. The turbine parameters summary is given in Table 1.

\section{Wind Turbine Control Strategy in the Nominal Production}

The nominal controller is designed for two objectives. On the one hand, the rotor speed should be regulated despite the wind changes. On the other hand, the blade's deflections should be attenuated to prevent the structure from being damaged. The control scheme is summarized in Figure 3. The blades' deflection velocity and the rotor speed are both measured and fed to the robust state observer. Finally, the 
TABLE 1: Wind turbine parameters.

\begin{tabular}{lc}
\hline Characteristics & Value \\
\hline Rated power $(\mathrm{MW})$ & 1.5 \\
$M_{b}:$ Blade mass $(\mathrm{kg})$ & 4,230 \\
$D_{b}:$ Blade stiffness $(\mathrm{N} \cdot \mathrm{m} / \mathrm{rad})$ & 20760 \\
Total rotor mass $(\mathrm{kg})$ & 32,016 \\
$J_{r}:$ rotor inertia $\left(\mathrm{kg} \cdot \mathrm{m}^{2}\right)$ & $2.9624 * 10^{6}$ \\
$J_{\mathrm{g}}:$ generator inertia $\left(\mathrm{kg} \cdot \mathrm{m}^{2}\right)$ & $53.036 * \mathrm{Ng}^{2}$ \\
$N_{g}:$ gearbox ratio & 87.965 \\
$R:$ rotor radius $(\mathrm{m})$ & 35 \\
\hline
\end{tabular}

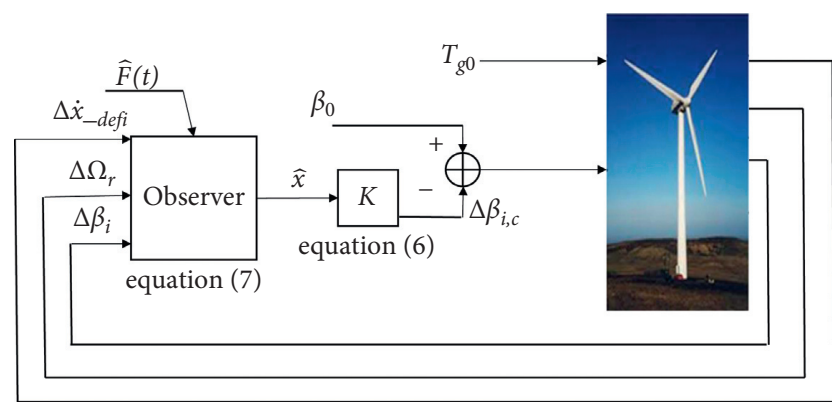

Figure 3: The wind turbine control objective scheme.

state feedback controller uses the estimated states to generate the necessary pitch angle variations responsible for regulating rotor speed and attenuating blade deflections. The adjective nominal means the baseline or the fault free situation. The system is linearized about the nominal operating point, and then the nominal controller is derived based on the pole placement method to achieve the nominal production. The opposite to nominal is faulty, which means the situation in which the fault occurs.

3.1. The Global Wind Turbine State Space Model. The global state space representation in the equation (5) takes into account the pitch actuator faults. The actuator effectiveness fault is modeled as a multiplication of the input matrix $B$ by an unknown fault matrix $F$, and the faulty system is then given by

$$
\left\{\begin{array}{l}
\dot{x}(t)=A x(t)+B F(t) u(t)+D_{1} \omega(t) \\
y(t)=C x(t)+D_{2} \omega(t)
\end{array}\right.
$$

where $x(t) \in \mathbb{R}^{n}$ is the state vector; $u(t) \in \mathbb{R}^{m}$ is the input vector; $y(t) \in \mathbb{R}^{p}$ is the measured output with $p \geq m$; and $C$ and $B$ have full $\operatorname{rank}(\operatorname{rank}(C)=p$ and $\operatorname{rank}(B)=m)$. $F(t) \in \mathbb{R}^{m \times m}$, and $F(t)=\operatorname{diag}\left(f_{1}(t), f_{2}(t), \ldots, f_{m}(t)\right)$ is the vector of faults on the actuators.

3.2. The Observer and the Baseline Controller Design. The three-blade flap deflection amplitude reduction and the rotor speed regulation are performed through a pole placement state feedback [39] as in equation (6). In fact, the nominal control law in equation (6) is based on the fault free (nominal) situation, in which the fault matrix $\mathrm{F}(\mathrm{t})$ equals the identity matrix which is constant.

$$
u=\Delta \beta_{i_{-}}=K \hat{x} .
$$

The variable $\hat{x}$ in equation (6) is the state vector to estimate. The gains of controller $K$ are design parameters to achieve the multiobjective wind turbine control. The variable $\hat{x}$ should be estimated robustly in the next paragraph.

In Figure 3, $T_{g 0}$ is the generator torque input at the operating point and is kept constant in the region of high winds, $\beta_{0}$ is the pitch angle input at the operating point, $\Delta \beta_{i_{-} c}$ is the pitch angle input variations from the operating point $(i=1,2,3), K$ is the control gain, $\Delta \dot{x}_{\text {def }_{-} i}$ are the deflection velocity variations from the operating point $(i=1$, 2, 3), $\Delta \Omega_{r}$ is the rotor speed variations from the operating point, $\Delta \beta_{i}$ is the measured pitch angles and are input to the state observer, and $F(t)$ is the fault matrix.

3.2.1. The Robust State Observer. The structure of the state observer is given by system (7) and illustrated by Figure 4:

$$
\dot{\hat{x}}(t)=A \widehat{x}(t)+B F(t) u(t)-L(C \widehat{x}(t)-y(t)),
$$

where $y(t)$ corresponds to the vector containing the measured deflection velocities $\Delta \dot{x}_{-} \operatorname{def}_{i}$ and rotor speed $\Delta \Omega_{r}$. The vector $u(t)$ corresponds to the measured three blade pitch angles. $F(t)$ is the matrix of the faults. $\widehat{x}(t)$ is the output of the observer.

$\hat{x}(t) \in \mathbb{R}^{n}$ are the observer states, $\hat{y}(t) \in \mathbb{R}^{n}$ are the observed outputs, $\widehat{F}(t) \in \mathbb{R}^{m \times m}$ is the estimate of $F(t)$, and $L(t) \in \mathbb{R}^{n \times p}$ is the estimator gain to be designed.

3.2.2. The Fault Matrix Estimator. The fault matrix $F(t)$ has to be estimated. This estimate is $\widehat{F}(t) \in \mathbb{R}^{m \times m}$ and has the following diagonal form: $\widehat{F}(\mathrm{t})=\operatorname{diag}\left(\widehat{f}_{1}, \widehat{f}_{2}, \widehat{f}_{3}\right)$, where $\widehat{f}_{i}$ is the estimation of the pitch fault on the actuator of index $i$. The following integrator-based algorithm is proposed:

$$
\dot{\hat{f}}_{i}=-\xi_{i} r_{i} e_{y}(t) u_{i}(t)
$$

where $i=1,2,3 ; \Xi=\operatorname{diag}\left(\xi_{1}, \xi_{2}, \ldots, \xi_{m}\right) ; \Xi \in \mathbb{R}^{m \times m}$ is called the learning rate; and $r_{i} \in \mathbb{R}^{1 \times p}$. The parameter $p$ is the number of measurements. $u_{i}(t)$ is the control input in the direction to the pitch actuator number $i$.

The adaptability of the estimation consists in the update of the fault estimate dynamics with time by acting on the learning rate $\Xi$ or the matrix $R=\left[\begin{array}{lll}r_{1} & r_{2} & r_{3}\end{array}\right]^{T}$. The matrix $R$ is an additional degree of freedom that can be used to refine the approximation $B^{T} P=R C$ in equation (15) in Section 3.2.3. An application of this matrix could be to refine equation (15) only in the direction of a selected measurement from matrix $C$ for a fixed $\eta$. For example, to refine equation (15) in the direction of the blade deflections, $R$ could be chosen equal to $\left[\begin{array}{cccc}0 & -470.4158 & 0 & 0 \\ 0 & 0.001 & -470.4158 & 0 \\ 0 & 0 & 0 & -470.4158\end{array}\right]$ and the resulting product $R C$ will then be equal to 


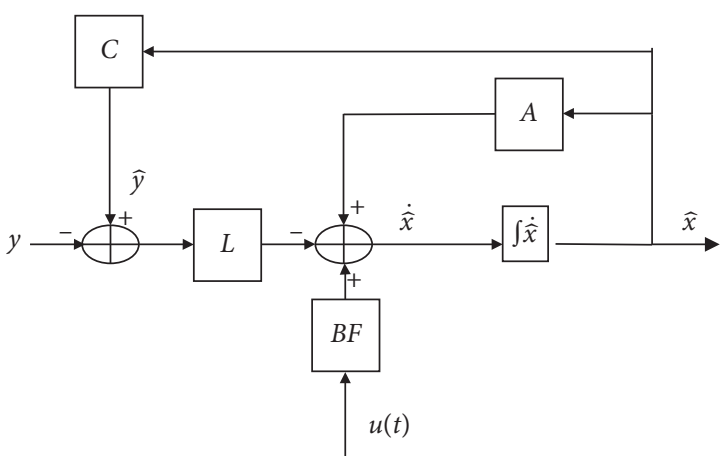

Figure 4: The state observer structure.

$\left[\begin{array}{ccccccc}0 & 0 & -470.4158 & 0 & 0 & 0 & 0 \\ 0 & 0 & 0 & 0 & -470.4158 & 0 & 0 \\ 0 & 0 & 0 & 0 & 0 & 0 & -470.4158\end{array}\right]$. In the resulting matrix $R C$, the second row vector became null. Indeed, this row represents the contribution of the rotor speed in the product matrix RC. The remaining vector rows 3,5 , and 6 represent the blade deflection velocities contribution in the product matrix RC. Another alternative could be to compute an initial guess of $R$ using YALMIP and refine it according to the need. In the present paper, $R$ was extracted using YALMIP.

The learning rate $\Xi$ is selected and handled by the user to ensure the fault estimation performance requirements. It allows to achieve a compromise between a quick fault accommodation and the disturbance amplification. Indeed, for high values of $\Xi$, high frequency information, namely, disturbances, is integrated in the learning process and vice versa. The variable $e_{y}(t)$ is the output tracking error and is equal to the difference $\hat{y}(t)-y(t)$.

3.2.3. Stability and Robustness of the Observer. Consider the states tracking error $e_{x}(t)=\widehat{x}(t)-x(t)$, the output tracking error $e_{y}(t)=\hat{y}(t)-y(t)$, and the fault tracking error $e_{f}(t)=\widehat{F}(t)-F(t)$.

The states' tracking error dynamics are given by the state space representation in the following equation:

$$
\left\{\begin{array}{l}
\dot{e}_{x}(t)=(A-L C) e_{x}(t)+B e_{f}(t) u(t)+\left(L D_{2}-D_{1}\right) \omega(t), \\
e_{y}(t)=C e_{x}(t)-D_{2} \omega(t) .
\end{array}\right.
$$

The first objective is to have $e_{x}(t) \longrightarrow 0$ and $e_{f}(t) \longrightarrow 0$ when $t \longrightarrow+\infty$. The tracking errors' convergence and stability could be ensured using the Lyapunov function $V_{e}(t)$ :

$$
V_{e}(t)=e_{x}^{T}(t) P e_{x}(t)+\operatorname{tr}\left(e_{f}^{T}(t) \Xi^{-1} e_{f}(t)\right) .
$$

The condition of validity of $V_{e}(t)$ is that $\Xi$ is chosen invertible.

$P$ is a symmetric positive definite matrix. The idea of the Lyapunov function choice is as the learning rate increases, the quadratic fault tracking error $e_{f}(t)$ decreases and the fault estimate tends to the actual fault. Finally, as the $P$ matrix decreases, the quadratic state tracking error $e_{y}(t)$ decreases and tends to the null vector.

The second objective is to minimize the effect of the wind turbulence on the output tracking error $e_{y}(t)$, using a performance index $\gamma>0$ as

$$
\left\|e_{y}(t)_{2}\right\| \leq \gamma\left\|\omega(t)_{2}\right\| .
$$

The minimization of the wind turbulence $\omega(t)$ effect on the output error could be performed using the following objective function:

$$
J=\int_{t_{f}}^{\infty} \frac{1}{\gamma}\left[e_{y}^{T}(t) e_{y}(t)-\gamma \omega(t)^{T} \omega(t)\right] \mathrm{d} t .
$$

The considered fault is time invariant. This means that $\dot{F}(t)=0$ and then $\dot{e}_{f}(t)=\dot{\vec{F}}(t)$. The derivative of $V_{e}(t)$ with respect to time is given by

$$
\begin{aligned}
\dot{V}_{e}(t)= & e_{x}^{T}(t)\left(P(A-L C)+(A-L C)^{T} P\right) e_{x}(t) \\
& +2 e_{x}^{T}(t) P B e_{f}(t) u(t)+2 e_{x}^{T}(t) P\left(L D_{2}-D_{1}\right) \omega(t) \\
& +2 \operatorname{tr}\left(e_{f}(t) \Xi^{-1} \dot{\widehat{F}}(t)\right) .
\end{aligned}
$$

Writing equation (8) as a matrix form gives the following:

$$
\Xi^{-1} \dot{\widehat{F}}=R e_{y}(t) u^{T}(t)=R C e_{x}(t) u^{T}(t)
$$

To eliminate the term containing both $e_{x}^{T}(t)$ and $u(t)$, equation (15) could be adopted. Indeed, by adopting (15) the second and fourth term in the development of the derivative of the Lyapunov cancel each other.

$$
B^{T} P=R C \text {. }
$$

In order to solve the condition (15), its LMI version is used as in the following lemma.

Lemma 1 (see [40]). The condition $B^{T} P=R C$ has an $L M I$ equivalent writing:

$$
\text { minimize } \eta \text { subject to }\left[\begin{array}{cc}
\eta I_{r} & B^{T} P-R C \\
* & \eta I_{n}
\end{array}\right]>0,
$$

where $\eta$ is a dimensionless constant used to approximate $B^{T} P=R C$ as an LMI. The smaller $\eta$ is, the accurate $B^{T} P=$ $R C$ is in LMI writing.

In the sequel, the symbol ' $*$ ' means the transposition of the term in the same diagonal as "*." For example, in equation (16), “*” is the transposition of the term $B^{T} P-R C$.

The derivative of the Lyapunov function becomes

$$
\begin{aligned}
\dot{V}_{e}(t)= & e_{x}^{T}(t)\left(P(A-L C)+(A-L C)^{T} P\right) e_{x}(t) \\
& +2 e_{x}^{T} P\left(L D_{2}-D_{1}\right) \omega(t) .
\end{aligned}
$$

By replacing the Lyapunov function derivatives in the cost function expression, the following inequality is obtained: 


$$
\begin{aligned}
J \leq & \int_{t_{f}}^{\infty} \frac{1}{\gamma} e_{y}^{T}(t) e_{y}(t)-\gamma \omega(t)^{T} \omega(t) \mathrm{d} t+\dot{V}_{e}(t) \\
= & \int_{t_{f}}^{\infty} \frac{1}{\gamma}\left[e_{y}^{T}(t) e_{y}(t)-\gamma \omega(t)^{T} \omega(t)\right] \mathrm{d} t+e_{x}^{T}(t)(P(A-L C) \\
& \left.+(A-L C)^{T} P\right) e_{x}(t)+2 e_{x}^{T} P\left(L D_{2}-D_{1}\right) \omega(t) .
\end{aligned}
$$

There is no direct action of the wind on the outputs; then, $D_{2}=0$. The objective function inequality:

$$
\begin{aligned}
J \leq & \frac{1}{\gamma} e_{y}^{T}(t) e_{y}(t)-\gamma \omega^{T}(t) \omega(t) \mathrm{d} t-2 e_{x}^{T}(t) P D_{1} \omega(t) \\
& +e_{x}^{T}(t)\left(P(A-L C)+(A-L C)^{T} P\right) e_{x}(t) \\
= & \int_{t_{f}}^{\infty} \zeta(t)^{T} \Upsilon \zeta(t) \mathrm{d} t
\end{aligned}
$$

where

$$
\begin{aligned}
\zeta(t) & =\left[\begin{array}{c}
e_{x}(t) \\
\omega(t)
\end{array}\right], \\
\Upsilon & =\left[\begin{array}{cc}
P(A-L C)+(A-L C)^{T} P+\frac{1}{\gamma} C^{T} C & -P D_{1} \\
* & -\gamma I_{d}
\end{array}\right] .
\end{aligned}
$$

Finally, to ensure the stability of the states' tracking error dynamics and the minimization of the wind turbulence on the output tracking error, the following inequality should hold:

$$
\Upsilon<0 \text {. }
$$

Using the Schur complement, the same previous inequality becomes

$$
\left[\begin{array}{ccc}
P A+A^{T} P-Y C-C^{T} Y^{T} & -P D_{1} & C^{T} \\
* & -\gamma I_{d} & 0 \\
* & * & -\gamma I_{p}
\end{array}\right]<0,
$$

where $Y=P L$, and then the error dynamic (9) satisfies the $H_{\infty}$ performance.

Remark 1. The adaptive algorithm (8) is proposed with the assumption that the input vector $u_{i}(t)$ satisfies the persistent excitation condition, which means that there exist positive scalars $\mu_{1}, \mu_{2}$, and $t_{0}$ such that for all $t$, the following inequality holds:

$$
\mu_{1} \leq \int_{t}^{t+t_{0}} u_{i}(s) u_{i}(s) d s \leq \mu_{2} .
$$

The poles of the designed observer could be placed through the linear matrix inequality in the following lemma.

Lemma 2 (see [41]). The eigenvalues of closed loop observed system dynamic matrix $(A-L C) \in \mathbb{R}^{n \times n} A \in R^{n \times n}$ belong to the circular region $D(\alpha, \tau)$ with center $\alpha+j 0$ and radius $\tau$ if and only if there exists a symmetric positive definite matrix $P \in \mathbb{R}^{n \times n} P \in R^{n \times n}$ such that the following condition holds:

$$
\left[\begin{array}{cc}
-P & P\left(A-L C-\alpha I_{n}\right) \\
* & -\tau^{2} P
\end{array}\right]<0 .
$$

The final integrated problem to be solved for the symmetric positive definite matrix $P$ is as the following lemma.

Lemma 3. Assume $D_{2}$ is null matrix and let a prescribed Ho performance level $\gamma$ be given. If there exists a symmetric positive definite matrix $P \in \mathbb{R}^{n \times n}$ and matrices $Y \in \mathbb{R}^{n \times p}$ and $R \in \mathbb{R}^{m \times p}$ such that the following condition holds:

$$
\begin{aligned}
& {\left[\begin{array}{ccc}
P A+A^{T} P-Y C-C^{T} Y^{T} & -P D_{1} & C^{T} \\
* & -\gamma I_{d} & 0 \\
* & * & -\gamma I_{p}
\end{array}\right]<0} \\
& {\left[\begin{array}{cc}
-P & P\left(A-L C-\alpha I_{n}\right) \\
* & -\tau^{2} P
\end{array}\right]<0,} \\
& \text { minimize } \eta \text { subject to }\left[\begin{array}{cc}
\eta I_{r} & B^{T} P-R C \\
* & \eta I_{n}
\end{array}\right]>0 \text {, }
\end{aligned}
$$

where $Y=P L$, then the error dynamic (9) satisfies the Ho performance and the poles of the robust state observer are within the region $D(\alpha, \tau)$.

3.2.4. The State Observer and Fault Simulation Results. The natural frequency of the blade is $f_{n}=(1 / 2 \pi)$ $\sqrt{\left(D_{b} / M_{b}\right)}=1.511 \mathrm{~Hz} . M_{b}$ is the blade mass and $D_{b}$ is the stiffness of the blade. It gives a natural pulsation of $\omega_{n}=9.49 \mathrm{rad} / \mathrm{s}$. All the frequencies above the natural frequency should be filtered by the observer. However, the observer should amplify the gain corresponding to $\omega_{n}$ for the estimation of the blades' deflections. The Bode diagrams of the designed observer in the system (7) are illustrated in Figures 5(a) and 5(b). The considered channel links the pitch actuator's input $\left(u_{1}(t)=\Delta \beta_{1}\right.$ in Figure 4) with the blade deflection observed state $\left(y_{3}(t)=\Delta x_{\text {def }_{-} 1}\right.$ in Figure 4$)$.

Figures 5(a) and 5(b) illustrate the designed observer Bode diagram function of the performance level $\gamma$ corresponding to the system in equation (7). The Bode diagrams are divided into two main regions. The first region is about the natural pulsation of the blades $\omega_{n}=9.49 \mathrm{rad} / \mathrm{s}$. In this region, the magnitude presents maximal values, which means that the blades' deflections are perfectly observed. The second region is where the pulsations are less than the natural pulsation of the blades. As $\gamma$ becomes smaller (closer to values near 0 ), the gain of the observer is reduced and the low frequencies of the wind speed are not amplified. The advantage is to prevent penetrating the amplified low frequencies contained in the rotor speed to the fault estimation algorithm through the residual $e_{y}(t)$. The effect of the amplified low frequencies is reducing the convergence speed of the estimation algorithm in equation (8). As a conclusion, the convergence speed of the fault estimation could be tuned 


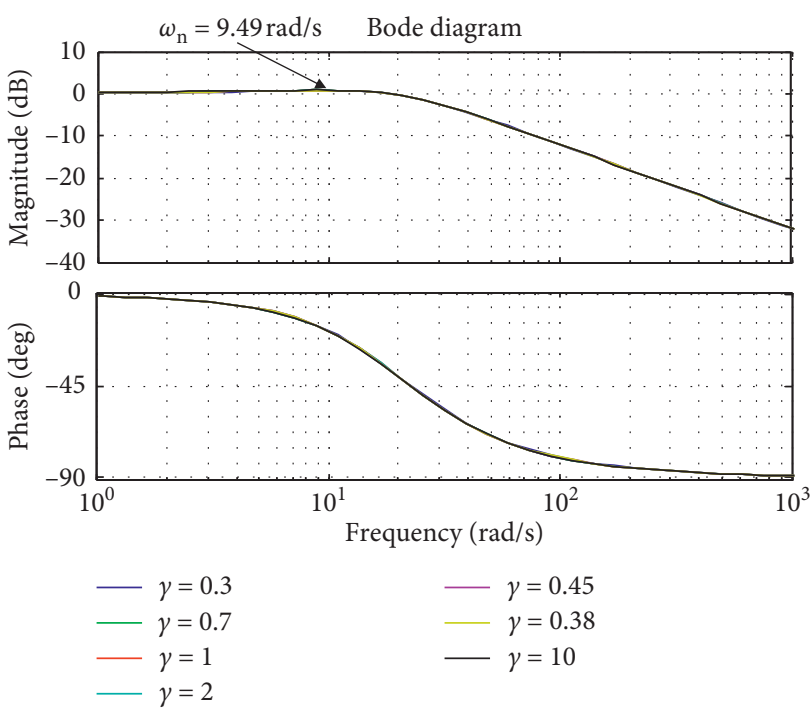

(a)

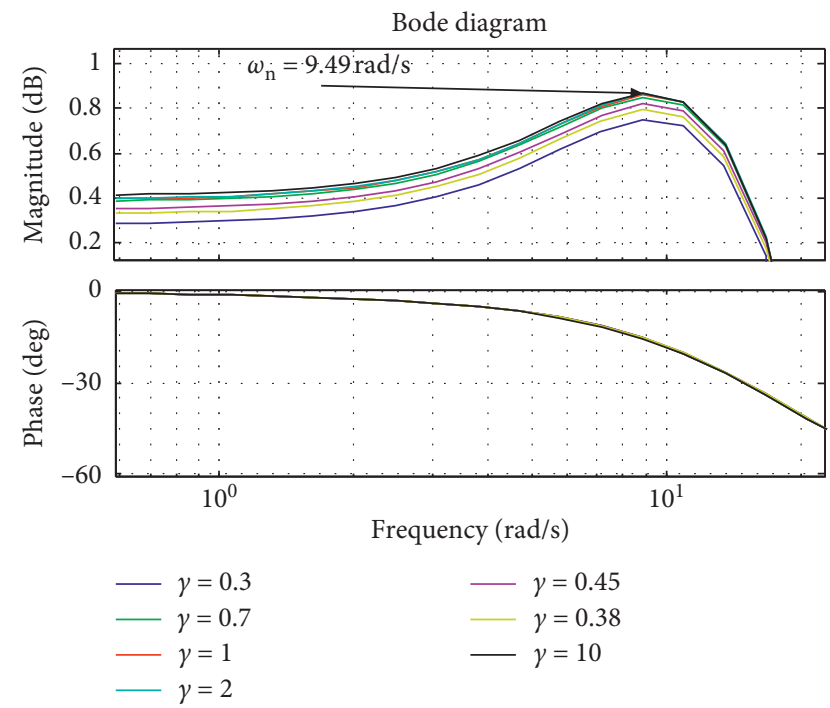

(b)

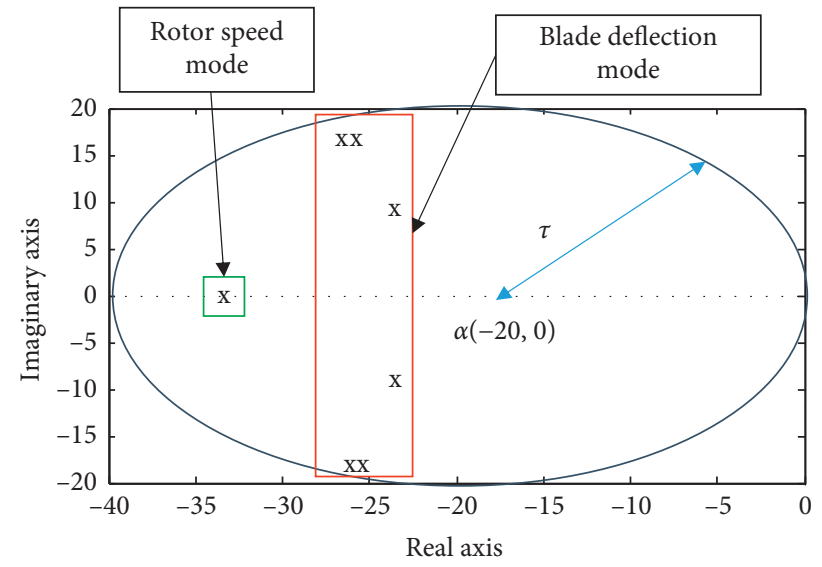

(c)

Figure 5: (a) Bode diagrams of the designed observer for different performance levels. (b) Zoom on the Bode diagrams of the designed observer for different performance levels. (c) The state observer pole placement for LMI region $(\alpha, \tau)=(-20,20)$ in the complex axis.

by the performance index $\gamma$ in addition to the tuning by the learning rate $\Xi$ as illustrated in Figure 6(a), where the learning rate is fixed at 0.1 and $\gamma$ takes the values 3,1 , and 0.3 .

Figures 7 and 8 illustrate the tracking errors in the faulty and the fault tolerant case for the rotor speed and the blade 1 deflection velocity as samples. The observation quality is defined by the states' tracking error. It could be noticed that a sufficient level of observation is achieved for $\gamma=0.3$ and $\xi_{i}=0.1(i=1,2,3)$.

For the rotor speed, in the fault tolerant case, the tracking error has a mean value of $-3.96 \times 10^{-4} \mathrm{rad} / \mathrm{s}$ and a standard deviation of $6.841 \times 10^{-4} \mathrm{rad} / \mathrm{s}$. For the faulty case, the mean value increases to $-0.002 \mathrm{rad} / \mathrm{s}$ with a standard deviation of $9.8893 \times 10^{-4} \mathrm{rad} / \mathrm{s}$. For the blade deflection velocity, in the fault tolerant case, the tracking error has a mean of $-0.058 \mathrm{~m} / \mathrm{s}$ and a standard deviation of $0.4973 \mathrm{~m} / \mathrm{s}$. For the faulty case, the mean value increases to $-0.0823 \mathrm{~m} / \mathrm{s}$ and a standard deviation of $0.6595 \mathrm{~m} / \mathrm{s}$.

Figure 5(c) illustrates the result of pole placement of the state observer. The chosen region is of center 20 and radius 20.
The resulting closed loop eigenvalues are $(-33.4089 ;-24$. $7069 \pm 8.6379 i ;-26.6204 \pm 18.7259 i ;-26.6204 \pm 18.7259 i)^{T}$.

In Figure 6(a), the effectiveness of actuator number 1 means $1-f_{1} . f_{1}$ represents the decrease in the output of the actuator.

In Figures 7 and 8 , the notations $e_{y 1}$ correspond to the rotor speed component and $e_{y 2}$ corresponds to the blade deflection velocity component in the tracking error vector $e_{y}$.

Figures 6(a) and 8 correspond to the first faulty scenario of Section 4 and Figure 7 corresponds to the third faulty scenario.

Figure 6(b) illustrates the fault estimation of the blade 1 actuator for several values of learning rate $\xi_{1}$. As the learning rate increases, the estimate of the fault converges rapidly to the actual fault (magenta).

Remark: There is a coupling effect between the fault estimation and the state observer due to the fault tolerant control (FTC) correction. Such an effect can be neglected as long as the state observer is slower than the fault estimation. This can be achieved by choosing properly the learning rate. 


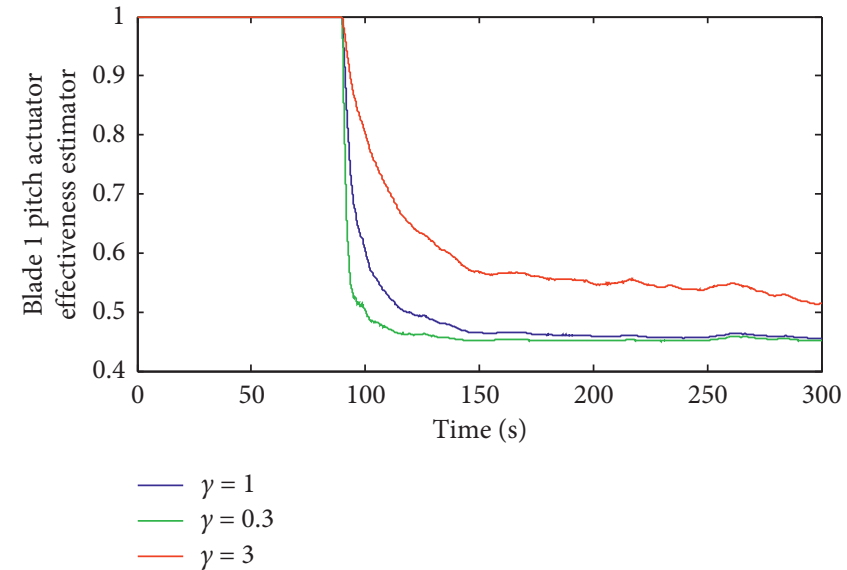

(a)

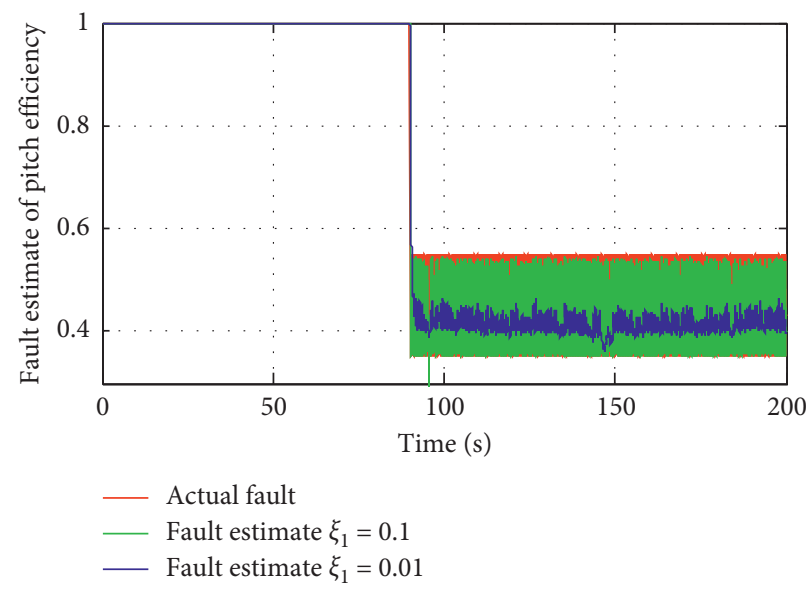

(c)

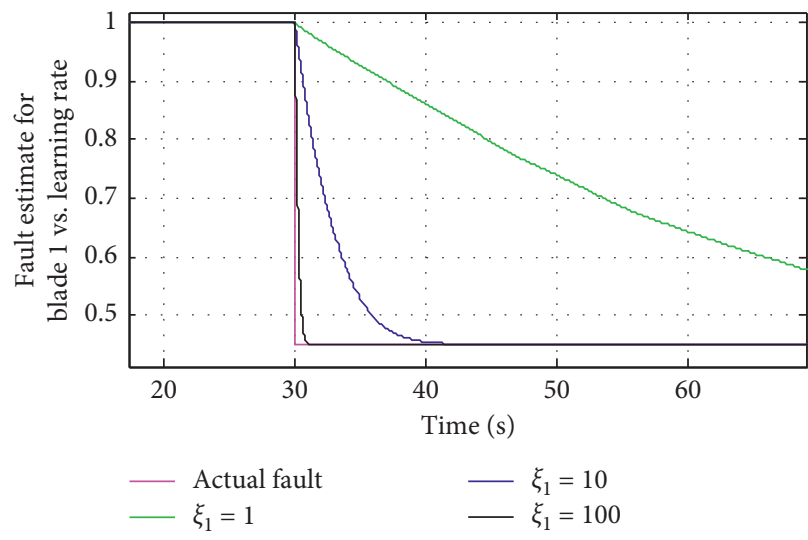

(b)

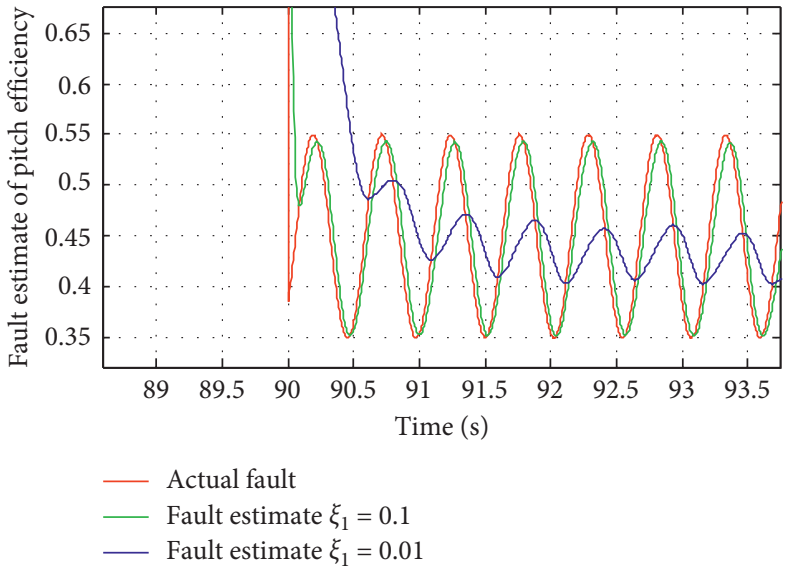

(d)

FIgURE 6: (a) Estimation of the pitch 1 actuator effectiveness for different performance levels. (b) Estimation of the pitch 1 actuator effectiveness for different learning rates. (c) Fault estimate with dynamics faster than system dynamics. (d) Zoom on fault estimate with dynamics faster than system dynamics.

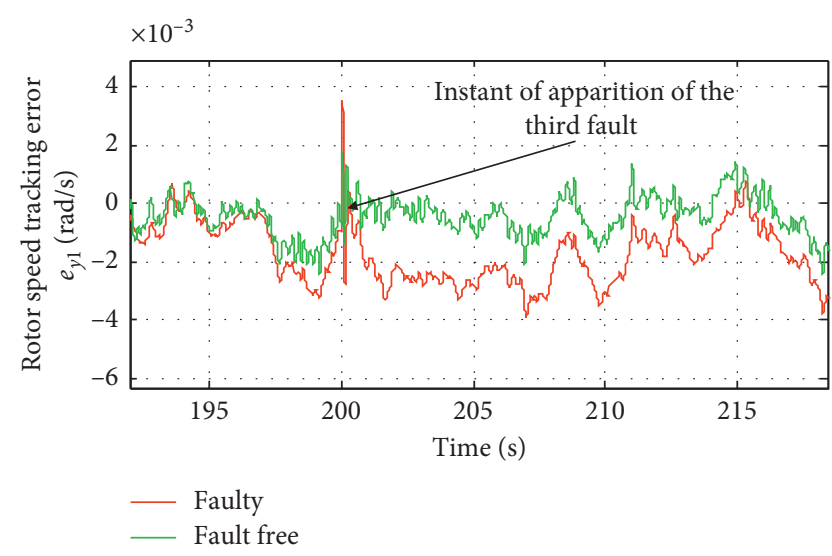

FIGURE 7: The rotor speed tracking error.

3.2.5. Fault Scenario with Dynamics Faster than the System Dynamics. In this section, a sinusoidal loss of efficiency is considered with frequency higher than $1.5 \mathrm{~Hz}$ of blades. Figures 6(c) and 6(d) show the result of fault estimation in that case. In the proposed strategy, by only tuning the learning

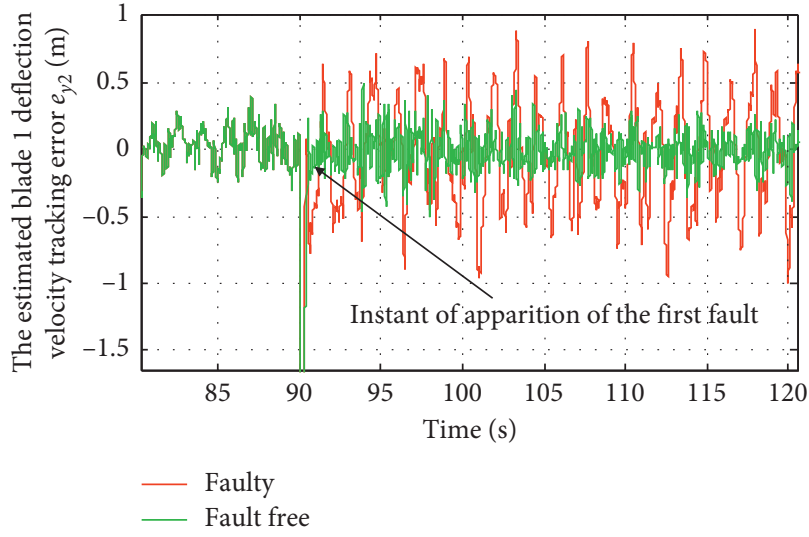

FIgURE 8: The estimated blade 1 deflection velocity tracking error.

rate to suitable higher values, the faults with dynamics faster than the system dynamics could be reconfigured without need to redesign the fault estimate algorithm as recommended in [33]. The amplitude of the fault is 0.1 and the frequency is $12 \mathrm{rad} / \mathrm{s}$ higher than $9.49 \mathrm{rad} / \mathrm{s}$ of the blades. 
In Figure 7, the most important issue is the change of the mean rotor speed error. This information is the only one needed by the algorithm about the fault on the rotor speed. In Figure 8, the change is obvious on the amplitude of the blades at the natural frequency $1.5 \mathrm{~Hz}$. This last information could be easily deduced by computing the value $1 /$ period. Indeed, in Figure 8, the period of the signals is equal to 0.66 seconds giving the blades a natural frequency of $1.5 \mathrm{~Hz}$.
In the faulty case, this increase in the mean and the standard deviation of the tracking error is integrated by the algorithm in equation (8) to deduce the fault amplitude.

3.2.6. The Baseline Controller Design. Initially, the eigenvalues of the dynamics matrix $A$ of the system are equal to the vector $\lambda_{0}$ as follows:

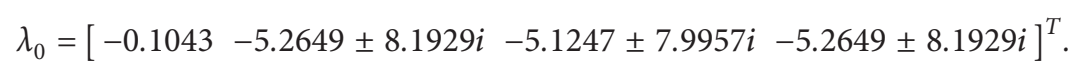

The eigenvalue -0.1043 corresponds to the rotor speed dynamic. The last three eigenvalues represent the three blades' dynamics. These eigenvalues are double conjugates because the blades represent an oscillatory system. The objective of the control is to reduce the oscillations of the blades by choosing new eigenvalues bigger in absolute value than the initial eigenvalues and with negative real parts to ensure stability of the closed loop. The design control parameter $K$ is computed so that the closed loop eigenvalues $\lambda_{(A+B K)}$ match the desired eigenvalues $\lambda_{d}$ :

$$
\lambda_{d}=\lambda_{(A+B K)} .
$$

In the present paper, the desired eigenvalues of the nominal closed loop are

$$
\lambda_{d}=\left[\begin{array}{lllllll}
-0.2 & -24.7 & -24.7 & -26 & -26 & -27.3 & -27.3
\end{array}\right]^{T} .
$$

The design steps of the nominal control are as follows:

(i) The controllability matrix of the pair $(A, B)$ is of full rank 7 . So, the system is controllable.

(ii) The control gain is defined as $K=\left(\begin{array}{lll}K_{11} & \ldots & K_{17} \\ K_{21} & \ldots & K_{27} \\ K_{31} & \ldots & K_{37}\end{array}\right)$. The gain $K$ is then substituted in the desired characteristic polynomial equation:

$$
\operatorname{det}\left(s I_{n}-A-B K\right)=\prod_{j=1}^{7}\left(s-\lambda_{\mathrm{d}, \mathrm{j}}\right)
$$

where $I_{n}$ is the identity matrix and $s$ is the Laplace operator. The integer $n$ is the number of states; it is equal to 7 in this paper. $\lambda_{d, j}$ is the $j$ th element of the desired closed loop eigenvalue vector $\lambda_{d}$.

(i) Equation (29) is solved by equating the identical powers on both sides. This allows extracting the value of the control matrix $K$.

Finally, the obtained control gain $K$ is applied to the system according to the scheme of Figure 3. The results of the regulation are depicted in Figures 9-12. Figures 9 and
10 illustrate that the speed regulation objective is achieved. In fact, as the rotor speed increases (decreases), the blade pitch angle increases (decreases) in order to prevent wind from driving the wind turbine (in order to provide more wind to drive the wind turbine). The expected achieved performance is of $90 \%$ of attenuation. In the open loop, a difference from the operating point of $10 \mathrm{rpm}$ at the instant $150 \mathrm{~s}$ is denoted. After applying the control strategy, the difference becomes $1 \mathrm{rpm}$, which corresponds to $90 \%$ of attenuation.

Figures 11 and 12 show that when the deflection increases (the red signal), the pitch action (green) increases to attenuate the deflection as presented in the same figure (blue signal). It can be concluded that the blade pitch action is composed of two signals: a low frequency control action responsible for rotor speed regulation and a high frequency control action responsible for mitigating the blade deflections.

Figures 9-12 correspond to the nominal case (healthy case). In this case, no fault is occurring.

\section{Wind Turbine Optimal Adaptive Fault Tolerant Control in the Faulty Operation}

The considered fault is the loss of efficiency in the three pitch actuators. The scenario created in the FAST software is a drop of $55 \%$ in the effectiveness of the pitch actuator 1 at the instant 90 s, $40 \%$ in the effectiveness of the pitch actuator 2 at the instant $150 \mathrm{~s}$, and $60 \%$ in the effectiveness of the pitch actuator 3 at the instant $200 \mathrm{~s}$.

4.1. The Fault Mechanism. The considered pitch actuator is hydraulic based. In Figure 13(a), the prefix $p$ denotes the piston side, the prefix $r$ denotes the rod side, and the prefix $v$ denotes the proportional valve side. $A_{p}$ and $A_{r}$ correspond, respectively, to the surfaces of the piston side and the rod side. The pump pumps oil from the accumulator with a pressure $p_{s}$ which passes across the proportional valve with a mass flow $Q_{v}$. Finally, the air arrives to the piston side with the pressure $p_{p}$ and thrusts the rod linked to the blades. The trust force is $F_{c y l}$ and is given by 


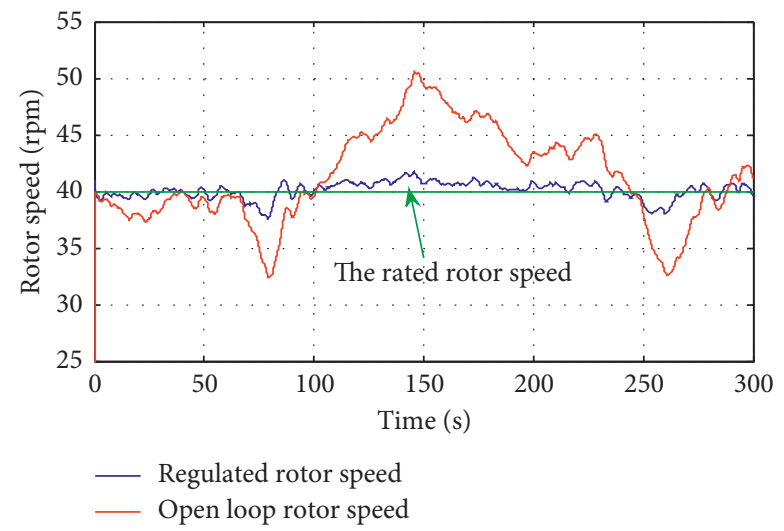

FIGURE 9: Rotor speed (rpm).

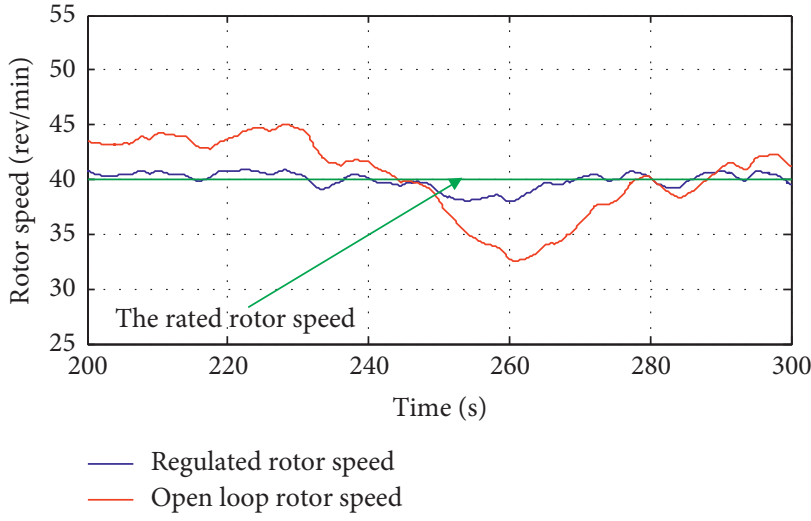

(a)

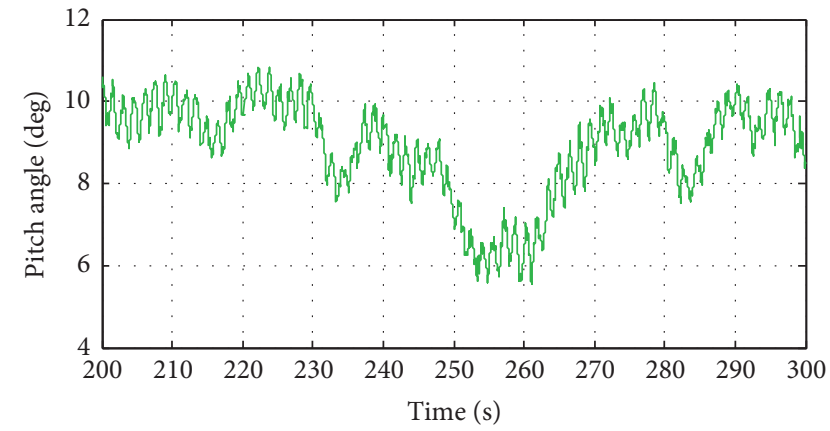

(b)

Figure 10: Zoom on the rotor speed (rpm) and the blade pitch angles (degrees) in the region [200, 300] sec.

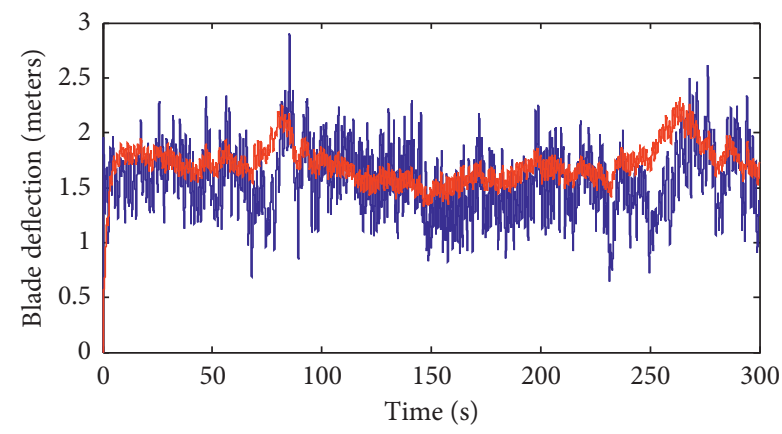

- Blade deflection without control

- Blade deflection after control

Figure 11: Blade deflection (meters).

$$
F_{\text {cyl }}=p_{p} A_{p}-p_{r} A_{r}-F_{\text {fric }}
$$

where $F_{\text {fric }}$ is the friction force of the piston on the cylinder and is linearly dependent to the displacement velocity of the piston.

For the same rod pressure $p_{r}$ and friction $F_{\text {fric }}$, the considered pitch loss of efficiency fault occurs when the pressure $p_{p}$ drops due to the ageing of the pump station. This ageing appears for example because of excessive Joule effect (heat) losses in the wirings of the pump motor. Under these conditions, the thrust force $F_{\text {cyl }}$ of the rod on the blade is no longer sufficient to actuate the blade. The thrust force $F_{\text {cyl }}$ is represented by the action matrix $B$ in equation (5). The fault is modeled as a loss of efficiency percentage (equal or less than $100 \%$ ) multiplied by the action matrix $B$. As a result, the pitch efforts are no longer sufficient for damping the oscillations of the blades. The aim of the algorithm in equation (8) is to track that multiplicative percentage with a sufficient precision and response time.

After that, equation (31) comes to amplify the supplied power (control) to the pump to compensate the lost energy due to Joule effect. The amplification is performed mathematically by dividing the old control signal by the estimated faulty efficiency.

4.2. The Fault Tolerant Controller. The fault tolerant control signal is obtained by dividing the initial baseline control signal by the estimated fault:

$$
u_{f}(t)=\widehat{F}(t)^{-1} u(t) .
$$

To avoid the division by zero in the case of zero efficiency, $\widehat{F}(t)$ should be replaced by a small number (for example, $\left.10^{-4}\right)$. The scheme of the global fault tolerant control is given by Figure 13(b). 

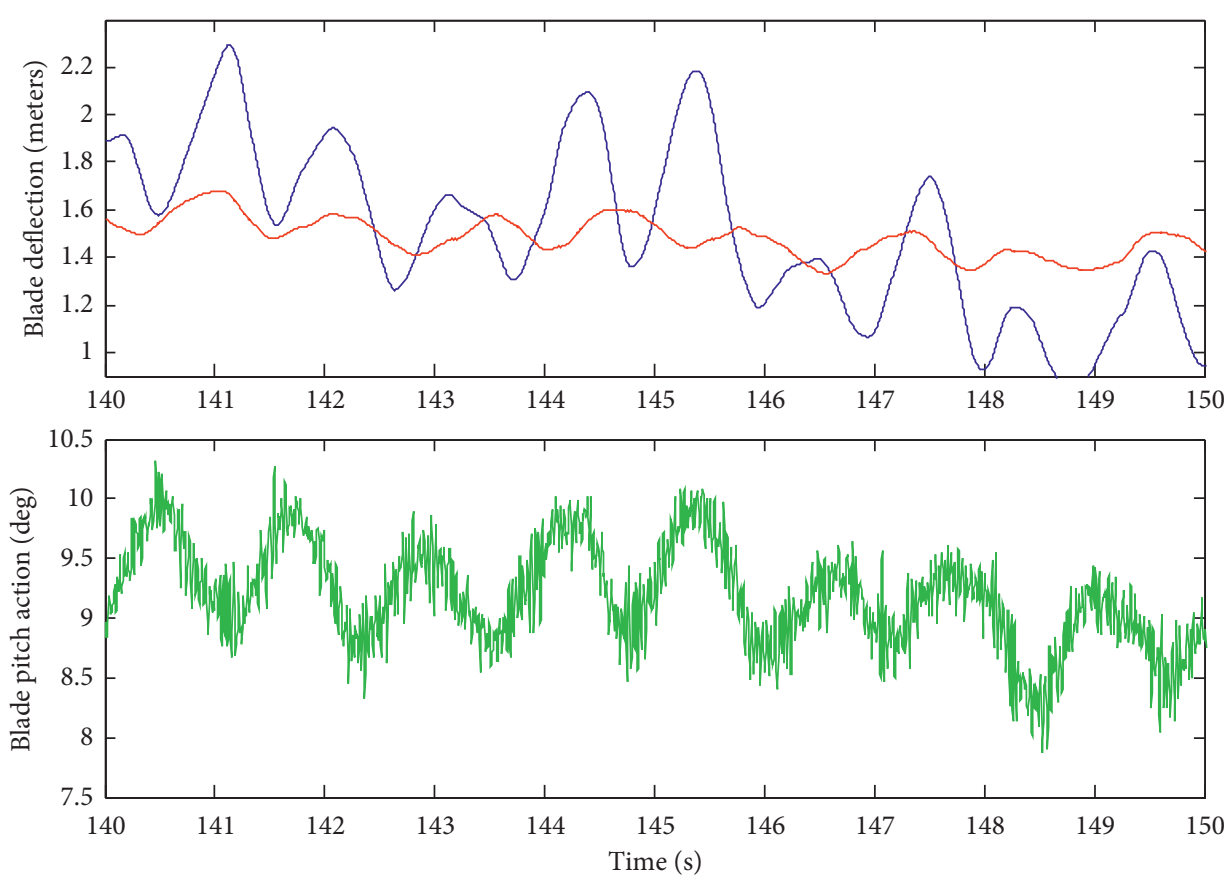

Without control

_ With control

Figure 12: Blade 1 deflection (meters) and blade 1 pitch action.

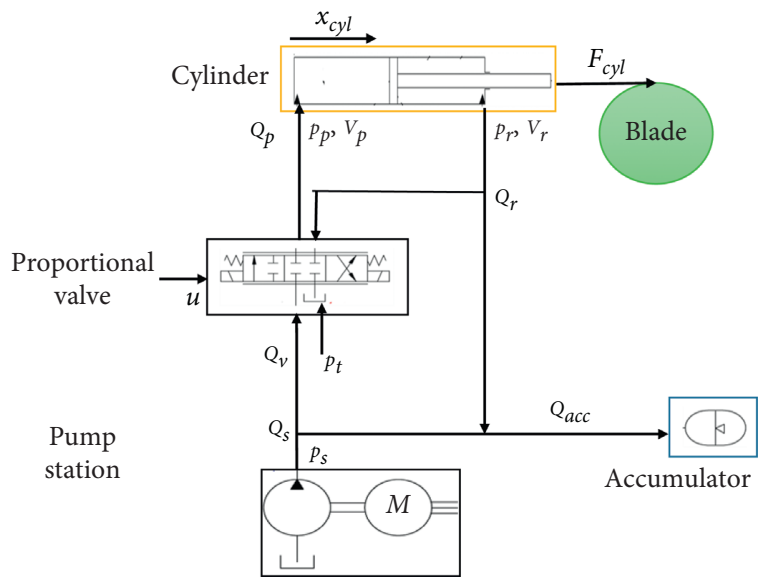

(a)

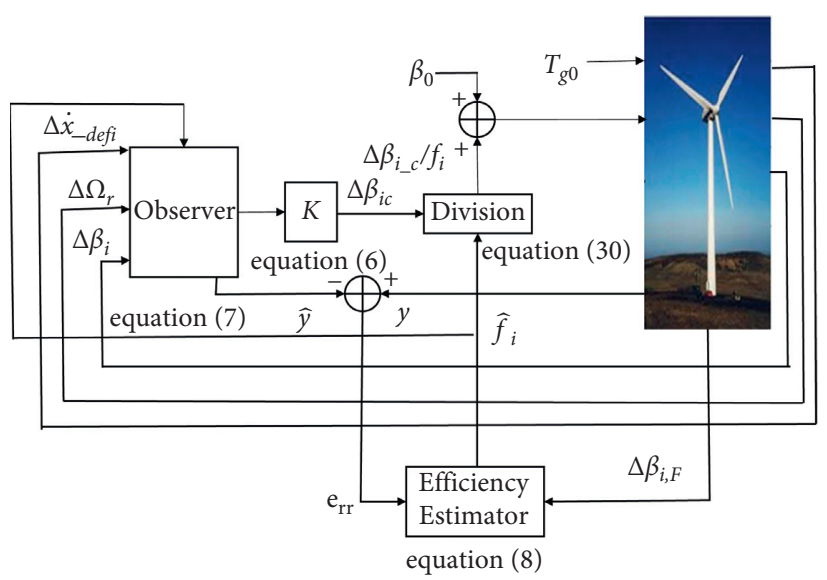

(b)

FIgURE 13: (a) The pitch actuator model. (b) The wind turbine control objective scheme.

Before being fed to the pitch actuator, the effectiveness is estimated by the adaptive algorithm. Then, the control signal is divided by the estimated efficiency to compensate the drop in the actual efficiency of the pitch actuator.

Remark: The different LMIs of the algorithm are resolved through the YALMIP tool [42].

$\Delta \beta_{i}$ is the pitch angle input variations from the operating point for the $i^{\text {th }}$ actuator $(i=1,2,3), \Delta \dot{x}_{-} \operatorname{def}_{i}$ are the deflection velocity variations from the operating point for the $i^{\text {th }}$ blade, and $\Delta \Omega_{r}$ is the rotor speed variations from the operating point.

$\Delta \beta_{i_{-} F}$ is the faulty pitch angle input variations from the operating point for each actuator, $\widehat{f}_{i}$ is the estimated efficiency for the $i^{\text {th }}$ actuator, and $\hat{x}$ is the estimated state variations of the wind turbine from the operating point.

4.3. The Three Pitch Actuators Loss of Effectiveness Faulty Scenario. Let us consider the blade without loss of generality. Figure 14 illustrates the blade 1 deflection.

In the faulty situation, the deflections increase from 1.5 meters to 4 meters because the pitch actuator could not answer completely to the corrective action of the baseline regulator against the wind. According to [43], the maximal allowable industrial deflection for the considered wind turbine is of $3 \mathrm{~m}$. In the faulty case, this limit is exceeded which increases the risk of damage of the blade. 
After applying the fault tolerant control strategy, the blade deflections are perfectly reconfigured.

Let $F(s)$ be the effect of the pitch angle $i$ on the blade $i$ deflection. The effect of the strategy on the blades deflections is interpreted as an additional zero at the origin in the transfer function $F(s)$. In Figure 15, it can be noticed that when $s$ tends to zero, $s F(s)$ tends to zero. The reconfiguration time for each fault estimation of index $i$ is dictated by the product $\xi_{i} \times r_{i}$.

The rapidity of the algorithm depends on the learning rate $\Xi$ and the matrix $R$. As studied earlier in the paper, in addition to the learning rate $\Xi$, even the performance level $\gamma$ could influence the response time of the fault estimation through the low frequencies of the wind. In fact, for a given $\gamma$, a matrix $P$ depending on $\gamma$ could be found through the LMI in equation (22). Then, by using the LMI in equation (15), the matrix $R$ is obtained and used to compute the estimation $\widehat{F}(t)$ using equation (8). As a conclusion, the parameter $\gamma$ influences the response time of the algorithm through the matrix $R$.

The derivative form of the block between $\Delta \beta_{i_{-} c}$ and $F(s)$ in Figure 15 has a risk of turbulence amplification. For this reason, a study to choose the learning rate $\Xi$ and the performance level $\gamma$ function of the correlation between the tracking error $e_{y_{-} 2}(t)$ (blade 1 deflection velocity as an example) and the wind turbulence is proposed in the next section.

4.4. The Choice of $\gamma$ and $\Gamma$ Based on the Correlation with Turbulence and the Estimation Response Time Criteria. In this section, empirical boundaries are proposed for choosing the performance level $\gamma$ and the learning rate $\Xi$ based on two criteria. Namely, the correlation between the wind speed $\omega$ and the residual $e_{y}$ is considered. This variable is crucial to investigate because it could be amplified if decided to use the performance level $\gamma$ to tune the estimation response time. The second criterion is the rapidity of convergence which is important to ensure because only short times of convergence are recommended for a fast recovery of the fault.

4.4.1. The Correlation between the Wind Turbulence and the Residual $e_{y}$. The expression of the correlation of the two signals is given by

$$
\text { Corr }=\frac{E\left[e_{y} \times \omega\right]-E\left[e_{y}\right] E[\omega]}{S D_{e_{y}} \times S D_{\omega}},
$$

where $E[x]$ denotes the expectation of the signal $x$ and $S D_{x}$ denotes the standard deviation of the signal $x$. The correlation Corr belongs to the interval $[-1,1]$. It obeys to the weak and strong correlation. If the coefficient Corr $\in\left[\begin{array}{ll}-0.5 & 0\end{array}\right] \cup$ $\left[\begin{array}{ll}0 & 0.5\end{array}\right]$, it is called a weak correlation. If the coefficient Corr $\in\left[\begin{array}{ll}-1 & -0.5\end{array}\right] \cup\left[\begin{array}{ll}0.5 & 1\end{array}\right]$, it is called a strong correlation.

4.4.2. The Estimation Response Time of the Algorithm. The response time of the fault estimation algorithm in equation (8) for each actuator of index $i$ is given by

$$
v_{i}=\xi_{i} \times r_{i} \text {. }
$$

Many simulations have been made on the FAST software in order to derive empirical data on $v$ and Corr helping to choose the appropriate values of the two parameters $\gamma$ and $\Xi$. Then, an interpolation between data points has been performed to define mappings linking the tracking error and the wind disturbance.

Figures 16 and 17 show the evolution of the correlation and fault estimation response time with the performance level and the learning rate for the considered wind turbine in Table 1. From Figure 18, one can conclude that for $\gamma$ less than 1 , this parameter influences the correlation more than the response time of the algorithm. However, when $\gamma$ exceeds 2.5 , the correlation becomes more influenced also by $\Xi$ as $\gamma$ increases. For $\gamma$ less than 2.25, the response time of the algorithm is influenced by both $\Xi$ and $\gamma$. But, as $\gamma$ increases, only $\Xi$ influences the response time of the algorithm. In order to guarantee a fast reconfiguration of the system performance, one should choose a small response time of the algorithm by choosing a suitable pair $(\gamma, \Xi)$. However, one should guaranty that neither the resulting performance level nor the learning rate amplifies the correlation between the tracking error $e_{y}$ and the wind turbulence $\omega$.

For the last reason, the two parameters should obey to the following empirical law. For example, if $\gamma$ is in $[0.9,1.4]$ interval, $\Xi$ should not be in $[2.21,9.84]$ (zone 1 in Figure 18) to avoid high correlation, and with the same idea, if $\gamma$ is chosen in $[1.4,2.02], \Xi$ should not be in the interval [8.317, 9.84] (zone 2 in Figure 18).

An example of a choice of the pair $(\gamma, \Xi)$ leading to a fast and less correlated response time is indicated in Figure 19 by $(\gamma, \Xi)=(2.25,1.25)$.

4.4.3. Discussion with respect to Some Existing Methods. The proposed strategy is not complicated and uses an integral structure with one Lyapunov function to prove stability and convergence of both state estimation error and fault estimation error. The proposed approach uses a reduced number of measurements. Indeed, only the rotor speed and the individual blades' deflection velocity are needed. This reduces the information needs with respect to other techniques such as the one in [44] (requiring also the generator speed measurement). It does not either require the information about the presumed fault size as in [45].

The residual-based techniques in [45-47] deal only with fault detection tasks. Contrariwise, the proposed approach deals with both fault estimation and fault isolation suitable for active fault tolerant control strategies. Indeed, the individual deflection velocity measurement of each blade allows a natural isolation of the fault blade without the need of additional isolation methods. This method avoids the intrinsic delays of online least-squares methods, which could have a negative impact on the fault estimation response time.

The proposed strategy is equipped with a tuning method to avoid amplification of wind turbulence in the direction of the high learning rates. This issue is not sufficiently discussed in other works related to wind turbines.

Contrariwise to other strategies, the proposed method could deal with faults having dynamics faster than the system dynamics only by tuning the learning rate suitably. 


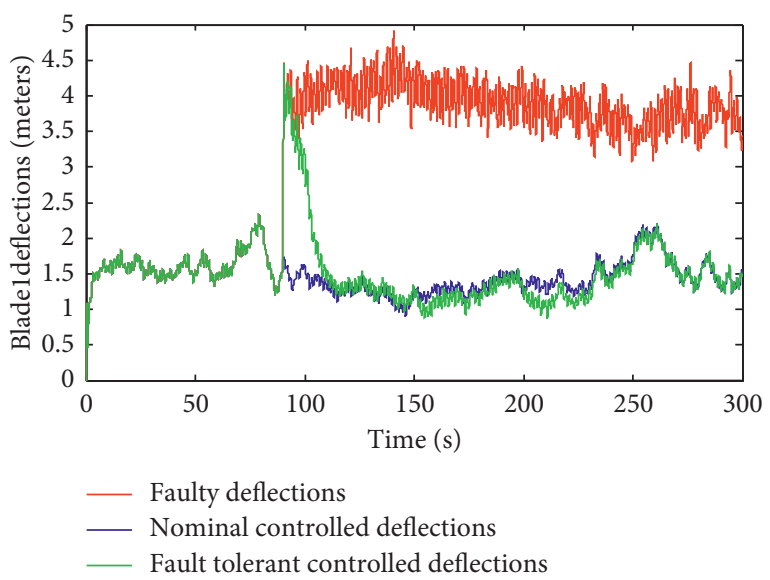

Figure 14: The bade deflections in the faulty deflections, nominal deflections, and fault tolerant deflections.

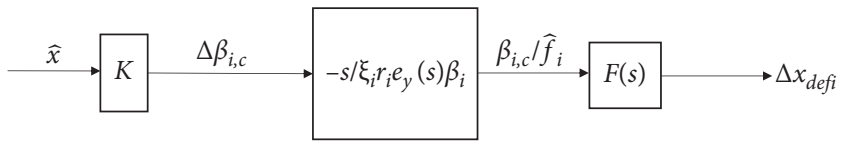

Figure 15: The effect of the reconfiguration on the blades.

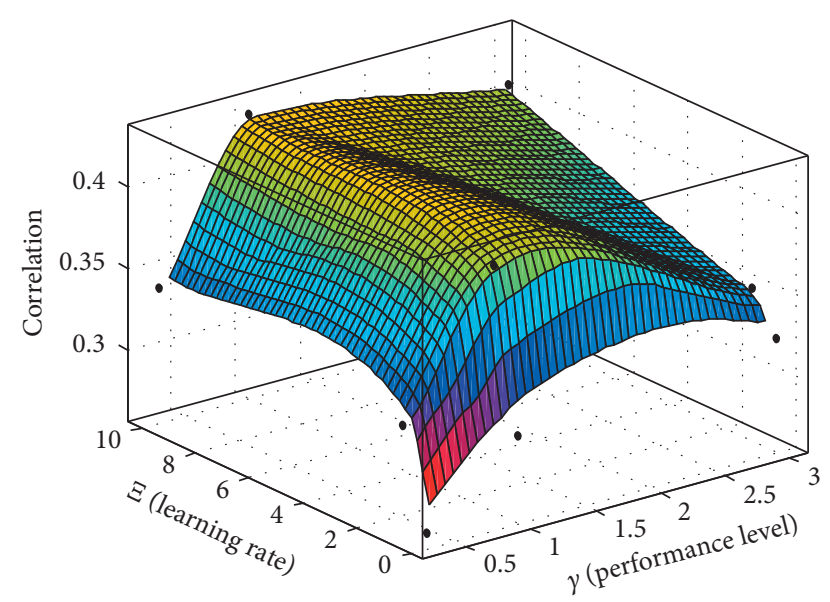

FIGURE 16: The correlation between measuring error and the turbulence with respect to the learning rate and the performance level.

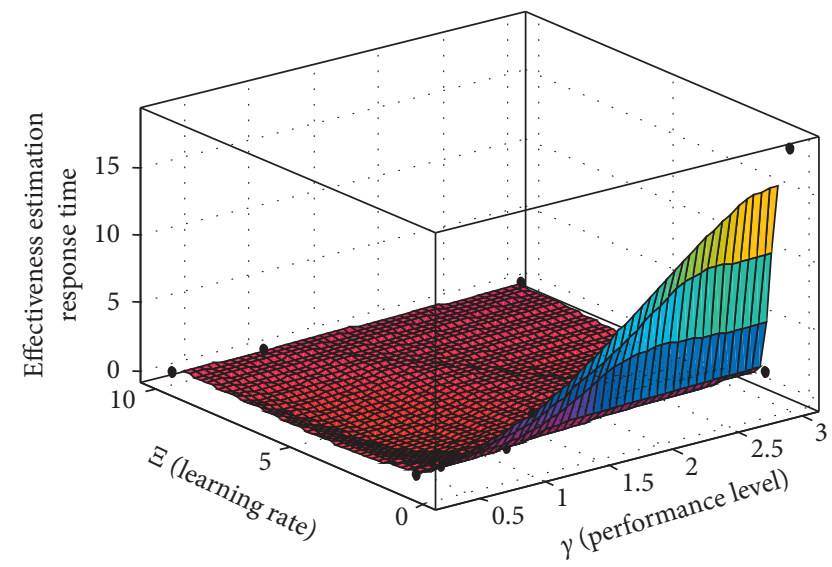

FIGURE 17: The effectiveness estimation response time with respect to the learning rate and the performance level.

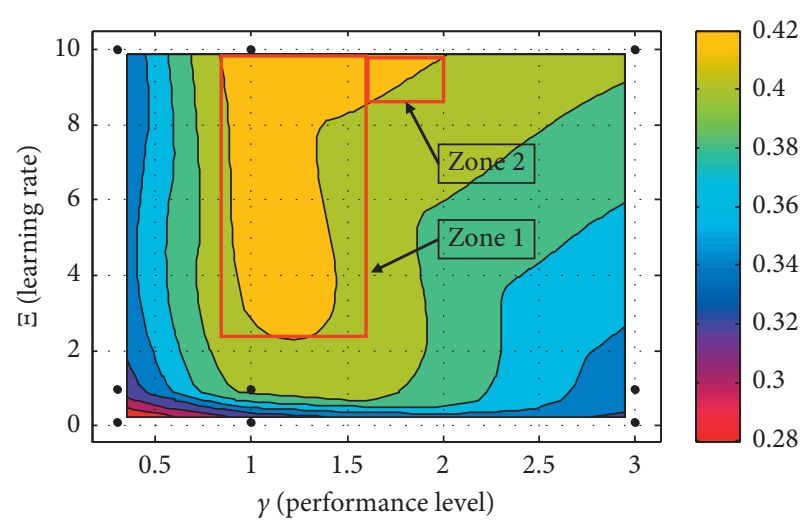

FIGURE 18: Maximal correlation zones vs. learning rate and performance level.

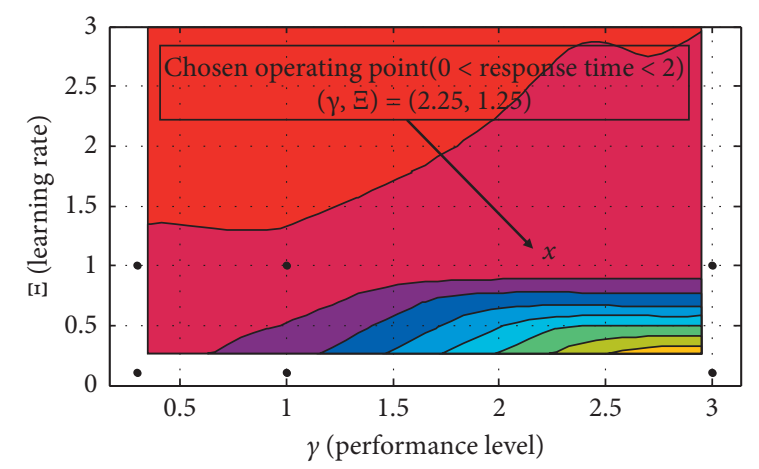

FIGURE 19: The effectiveness estimation algorithm response time vs. learning rate and performance level.

4.4.4. Comparison of the Proposed Strategy with the Virtual Actuator with State Residual Integrator. A new scenario of pitch fault on blade 1 is considered for the comparison between the proposed strategy and a virtual actuator-based strategy (details of this method are not the objective of the paper, [48]). In this section, the considered fault happend at the instant 15 seconds. This fault represents pitch actuator loss of efficiency of $55 \%$.

Figure 20 illustrates the structure of the strategy used for comparison. The principle of this strategy is to use a model reference and to compute the difference between the estimated faulty states and the model reference states. This difference is integrated and used as new control law. Equation (8) was used to derive the faulty matrix $B_{f}$. The difference with respect to the proposed strategy is in the integration of the state residual $x_{\Delta}$ instead of the division in equation (30) and the use of reference model.

Figure 21 illustrates a comparison between the proposed strategy and the virtual actuator with integrating the state residual in terms of blade fault reconfiguration. In some regions where the wind speed makes high amplitudes of high frequencies, which means the effort needed for integration is higher, the performance of the virtual actuator becomes poor in the interval $[16 s ; 26 s]$. This could be explained by the existence of two integrators, the first presented in equation (8) used to estimate the fault needed by $B_{f}$ and the second to integrate the state residual. In conclusion, the 


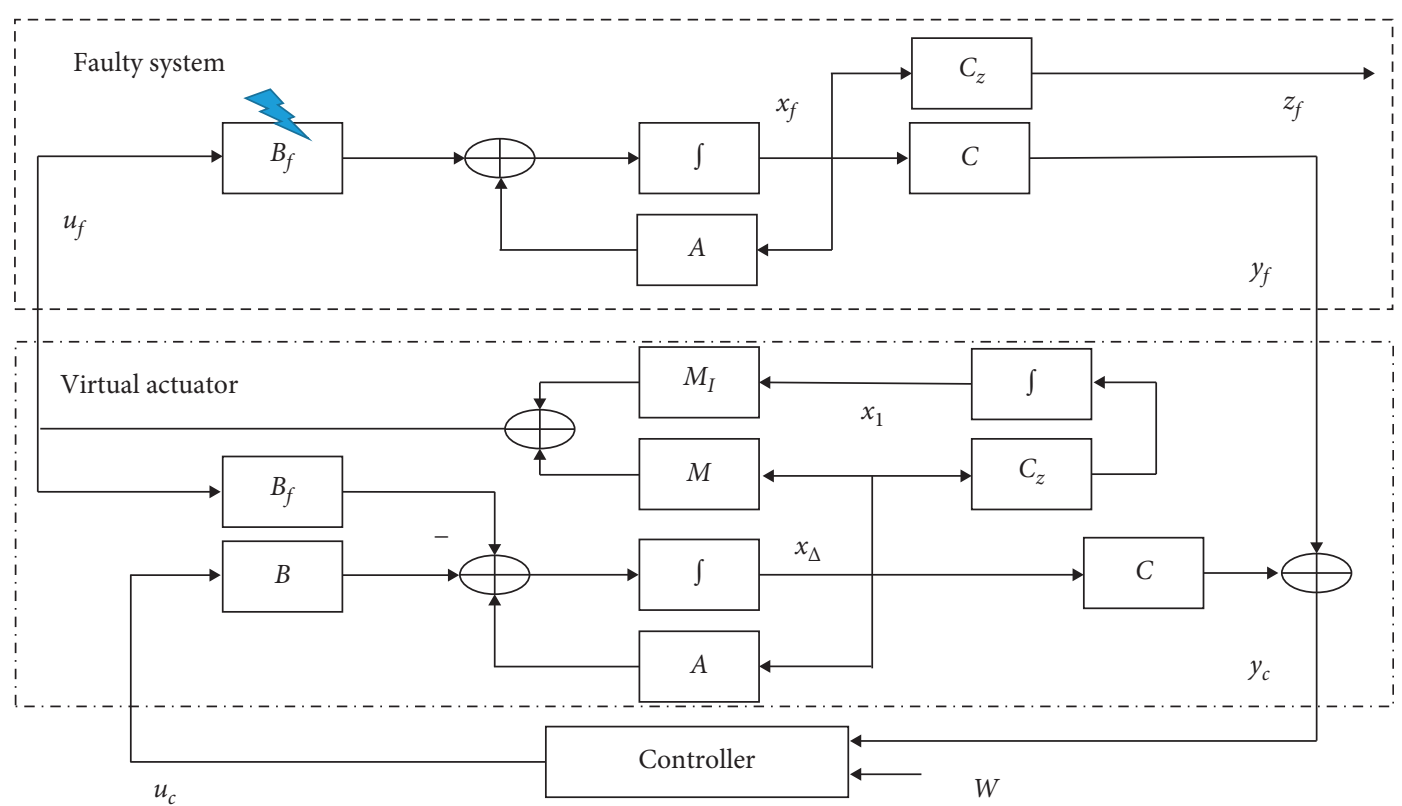

Figure 20: The simplified virtual actuator strategy with state residual $\mathbf{x}_{\Delta}$ integration.

virtual actuator with integration of state residual is not always suitable for integrator-based fault estimators.

4.4.5. Digital Implementation of the Strategy. The equations to be implemented are the baseline control law in equation (6), the observer in equation (7), and the fault estimation in equation (8). In order to digitalize the continuous equations, the first-order approximation in equation (34) is used. If $\dot{z}$ is the derivative, it could be approximated by

$$
\dot{z}(t)=\frac{z(t+T)-z(t)}{T},
$$

where $T$ is the sampling step. Hence, the strategy could be digitalized for each iteration of index $k$ as follows.

The digitalized baseline controller (from equation (6)) is given by

$$
u_{k}=K \widehat{x}_{k}
$$

The digitalized robust observer (from equation (7)) is given by

$$
x_{k+1}=A_{d} \widehat{x}_{k}+B_{d} F_{k} u_{k}+L_{d}\left(\widehat{y}_{k}-y_{k}\right)
$$

where $A_{d}, B_{d}$, and $L_{d}$ are, respectively, the state matrix, the input matrix, and the observer matrix. The expressions of the previous matrix are given by $A_{d}=I_{n}+T A ; B_{d}=T B$; and $L_{d}=T L$.

The digitalized fault estimation (equation (8)) for each actuator of index $i$ is given by

$$
f_{k+1, i}=f_{k, i}+T \xi_{k, i} r_{k, i} e_{y, k, i} u_{k, i} .
$$

\section{Conclusion}

In this paper, the partial failure of pitch actuators in wind turbine was considered. The LMI-based fault tolerant

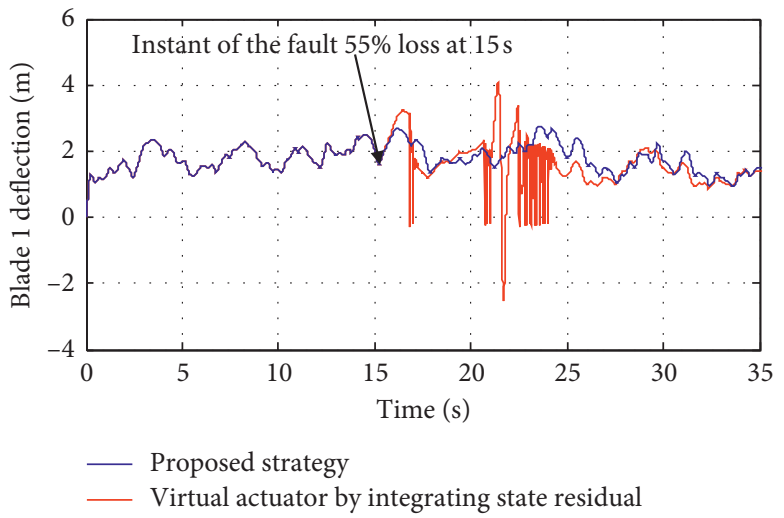

FIgURE 21: The comparison of the faulty blade 1 deflection between the proposed FTC and the residual integration FTC.

control was applied to the considered fault. The method presents the advantages that it estimates at the same time the states and the fault. The method convergence is proved through Lyapunov theory. Two key design parameters were identified in the integrated design, namely, the performance level and the learning rate. It has been shown that the performance level $\gamma$ of the observer and the learning rate $\Xi$ impact indirectly the loads on the turbine. The parameters have an effect on the availability time of the fault estimation. In some zones of the pair $(\gamma, \Xi)$, it has been found that as the learning rate increases, the fault becomes quickly available and the fault tolerant strategy accommodates rapidly the prefault load reduction. In other zones, the robustness $\gamma$ enhances the loads reduction. Moreover, the performance level $\gamma$ provides another degree of freedom to the adaptive algorithm in addition to the learning rate $\Xi$. An empirical design method was proposed to impose both a given correlation of the measuring error to the wind turbulence and a given response time of the effectiveness estimation algorithm. The proposed method helps choosing the desired short 
response time of the effectiveness estimation algorithm with sufficiently good correlation level to the wind turbulence.

\section{Appendix}

$$
\begin{aligned}
& A=\left[\begin{array}{ccccccc}
0.0328 & 0.1046 & 0.0087 & 0.1046 & 0.0087 & 0.1046 & 0.0087 \\
0 & 0 & 1 & 0 & 0 & 0 & 0 \\
-41.4716 & -91.8644 & -10.3888 & -1.6712 & -0.1391 & -1.6712 & -0.1391 \\
0 & 0 & 0 & 0 & 1 & 0 & 0 \\
-41.4712 & -1.6712 & -0.1391 & -91.8644 & -10.3888 & -1.6712 & -0.1391 \\
0 & 0 & 0 & 0 & 0 & 0 & 1 \\
-41.4721 & -1.6712 & -0.1391 & -1.6712 & -0.1391 & -91.8644 & -10.3888
\end{array}\right], \\
& \mathrm{B}=\left(\begin{array}{ccc}
0.0015 & 0.0015 & 0.0015 \\
0 & 0 & 0 \\
-2.0553 & -0.0234 & -0.0234 \\
0 & 0 & 0 \\
-0.0234 & -2.0553 & -0.0234 \\
0 & 0 & 0 \\
-0.0234 & -0.0234 & -2.0553
\end{array}\right) \\
& \mathrm{C}=\left(\begin{array}{lllllll}
1 & 0 & 0 & 0 & 0 & 0 & 0 \\
0 & 0 & 1 & 0 & 0 & 0 & 0 \\
0 & 0 & 0 & 0 & 1 & 0 & 0 \\
0 & 0 & 0 & 0 & 0 & 0 & 1
\end{array}\right) \\
& \mathrm{D}_{\mathrm{d}}=\left(\begin{array}{ccc}
0.0317 & 0.0317 & 0.0317 \\
0 & 0 & 0 \\
1.5187 & -0.5062 & -0.5062 \\
0 & 0 & 0 \\
-0.5062 & 1.5187 & -0.5062 \\
0 & 0 & 0 \\
-0.5062 & -0.5062 & 1.5187
\end{array}\right) ; \quad \gamma=0.3 ; \eta=1 \text {, } \\
& \Xi=\left(\begin{array}{ccc}
0.01 & 0 & 0 \\
0 & 0.01 & 0 \\
0 & 0 & 0.01
\end{array}\right) \\
& P=\left(\begin{array}{ccccccc}
0.0514 & -0.0001 & 0 & -0.0001 & 0 & -0.0001 & 0 \\
-0.0001 & 1.4086 & 0 & 0.1383 & 0 & -0.1618 & 0 \\
0 & 0 & 0 & 0 & 0 & 0 & 0 \\
-0.0001 & 0.1383 & 0 & 1.2979 & 0 & 0.0837 & 0 \\
0 & 0 & 0 & 0 & 0 & 0 & 0 \\
-0.0001 & -0.1618 & 0 & 0.0837 & 0 & 0.5037 & 0 \\
0 & 0 & 0 & 0 & 0 & 0 & 0
\end{array}\right), \\
& L=\left(\begin{array}{cccc}
34.3109 & 0.0237 & 0.0237 & 0.0237 \\
0.0021 & 1 & 0 & 0 \\
-429.7260 & 19.0461 & -0.4400 & -0.4399 \\
0.0012 & 0 & 0.9999 & 0 \\
-429.7430 & -0.4400 & 19.0465 & -0.4400 \\
0.0050 & 0 & 0 & 0.9999 \\
-429.7193 & -0.4399 & -0.4400 & 19.0463
\end{array}\right), \\
& R=\left(\begin{array}{cccc}
0.2114 & -157.5057 & 0.1955 & 0.1951 \\
0.1633 & 0.1955 & -157.5057 & 0.1953 \\
0.2212 & 0.1951 & 0.1954 & -157.5067
\end{array}\right) \text {, } \\
& K=10^{3}\left(\begin{array}{ccccccc}
-2.1347 & 4.3367 & 0.2346 & -2.3593 & -0.1786 & -2.5160 & -0.1820 \\
-0.0108 & 0.0142 & 0.0009 & 2.1925 & 0.1172 & -2.2166 & -0.1185 \\
0.0077 & 0.0283 & 0.0014 & -2.1936 & -0.1173 & 2.2145 & 0.1184
\end{array}\right) \text {. }
\end{aligned}
$$




\section{Data Availability}

No data were used to support this study.

\section{Conflicts of Interest}

The authors declare that they have no conflicts of interest.

\section{References}

[1] M. Ors, "Maximum power point tracking for small scale wind turbine with self-excited induction generator," Control Engineering and Applied Informatics, vol. 11, no. 2, pp. 30-34, 2009.

[2] N. A. Cutululis and E. Ceanga, "Robust control of an autonomous wind power system," Control Engineering and Applied Informatics, vol. 6, no. 4, pp. 3-12, 2004.

[3] H. P. Wang, A. Pintea, N. Christov, P. Borne, and D. Popescu, "Modelling and recursive power control of horizontal variable speed wind turbines," Control Engineering and Applied Informatics, vol. 14, no. 4, pp. 33-41, 2012.

[4] E. A Bossanyi, A. Kumar, and O. Hugues-Salas, "Wind turbine control applications of turbine-mounted LIDAR," Journal of Physics: Conference Series, vol. 555, 2012.

[5] C. Vlad, I. Munteanu, A. I. Bratcu, and E. Ceangă, "Anticipative control of low-power wind energy conversion systems for optimal power regime," Control Engineering and Applied Informatics, vol. 11, no. 4, pp. 26-35, 2009.

[6] T. K. Barlas, W. van Wingerden, A. W. Hulskamp, G. A. M. van Kuik, and H. E. N. Bersee, "Smart dynamic rotor control using active flaps on a small-scale wind turbine: aeroelastic modeling and comparison with wind tunnel measurements," Wind Energy, vol. 16, no. 8, 2012.

[7] E. A. Bossanyi, "Individual blade pitch control for load reduction," Wind Energy, vol. 6, no. 2, pp. 119-128, 2003.

[8] I. Houtzager, J. W. van Wingerden, and M. Verhaegen, "Wind turbine load reduction by rejecting the periodic load disturbances," Wind Energy, vol. 16, no. 2, pp. 235-256, 2013.

[9] P. J. Darrow, Wind Turbine Control Design to Reduce Capital Costs, Colorado School of Mines Golden, Golden, CO, USA, 2009.

[10] J. Ribrant and L. M. Bertling, "Survey of failures in wind power systems with focus on Swedish wind power plants during 1997-2005," IEEE Transactions on Energy Conversion, vol. 22, no. 1, pp. 167-173, 2007.

[11] T. Jain, J. J. Yame, and D. Sauter, "A novel approach to realtime fault accommodation in NREL's 5-MW wind turbine systems," IEEE Transactions on Sustainable Energy, vol. 4, no. 4, pp. 1082-1090, 2013.

[12] R.-E. Precup, P. Angelov, B. S. J. Costa, and M. SayedMouchaweh, "An overview on fault diagnosis and natureinspired optimal control of industrial process applications," Computers in Industry, vol. 74, pp. 75-94, 2015.

[13] J. Sá da Costa, B. M. S. Santos, and M. J. G. C. Mendes, “A design approach to FD/FTC of complex networked control systems," Control Engineering and Applied Informatics, vol. 3, no. 4, pp. 3-11, 2015.

[14] A. A. Ozdemir, P. Seiler, and G. J. Balas, "Wind turbine fault detection using counter-based residual thresholding," IFAC Proceedings Volumes, vol. 44, no. 1, pp. 8289-8294, 2011.

[15] J. M. Jonkman and M. L. Buhl, "Fast user's guide," 2005, https://nwtc.nrel.gov/fast7.

[16] J. M. Jonkman and M. L. Buhl, “TurbSim user's guide,” 2007, https://nwtc.nrel.gov/turbsim.
[17] L. Jong-Won, K. Joong-Kwan, H. Jae-Hung, and S. HyungKee, "Active load control for wind turbine blades using trailing edge flap," Wind and Structures, vol. 16, no. 3, pp. 263-278, 2013.

[18] E. E. Bachynski, M. Etemaddar, M. I. Kvittem, C. Luan, and T. Moan, "Dynamic analysis of floating wind turbines during pitch actuator fault, grid loss, and shutdown," Energy Procedia, vol. 35, pp. 210-222, 2013.

[19] V. Rezaei and F. R. Salmasi, "Robust adaptive fault tolerant pitch control of wind turbines," Wind Engineering, vol. 38, no. 6, pp. 601-612, 2014.

[20] P. F. Odgaard, J. Stoustrup, M. Kinnaert, and M. Kinnaert, "Fault-tolerant control of wind turbines: a benchmark model," IEEE Transactions on Control Systems Technology, vol. 21, no. 4, pp. 1168-1182, 2013.

[21] D. Belkhiat, D. Jabri, and I. Kilani, "Fault tolerant control for a class of switched linear systems using generalized switched observer scheme," Control Engineering and Applied Informatics, vol. 17, no. 4, pp. 90-101, 2015.

[22] R. Ebrahimi Bavili, M. J. Khosrowjerdi, and R. Vatankhah, "Active fault tolerant controller design using model predictive control," Control Engineering and Applied Informatics, vol. 17, no. 3, pp. 68-76, 2015.

[23] V. Rezaei and K. E. Johnson, "Robust fault tolerant pitch control of wind turbines," in Proceedings of the 52nd IEEE Conference on Decision and Control, Florence, Italy, December 2013.

[24] J. Kusnick, D. E. Adams, and D. T. Griffith, "Wind turbine rotor imbalance detection using nacelle and blade measurements," Wind Energy, vol. 18, no. 2, pp. 267-276, 2015.

[25] G. Ramtharan, N. Jenkins, O. Anaya-Lara, and E. Bossanyi, "Influence of rotor structural dynamics representations on the electrical transient performance of FSIG and DFIG wind turbines," Wind Energy, vol. 10, no. 4, pp. 293-301, 2007.

[26] Y. A. Elmaati, L. El Bahir, and K. Faitah, "An integrator based wind speed estimator for wind turbine control," Wind and Structures, vol. 21, no. 4, pp. 443-460, 2015a.

[27] X. Wei and M. Verhaegen, "Sensor and actuator fault diagnosis for wind turbine systems by using robust observer and filter," Wind Energy, vol. 14, no. 4, pp. 491-516, 2011.

[28] Y. A. Elmaati, L. E. L. Bahir, and K. Faitah, "Residual generation for the gearbox efficiency drop fault detection in the NREL 1.5 WindPact turbine," in Proceedings of the IEEE International Conference on Electrical and Information Technologies, Marrakech, Morocco, March 2015.

[29] E. Nabil, A. A. Sobaih, and B. Abou-Zalam, "Constrained Kalman filter based detection and isolation of sensor faults in a wind turbine," in Proceedings of the IEEE Tenth International Conference on Computer Engineering and Systems (ICCES), pp. 67-72, Cairo, Egypt, December 2015.

[30] Y. A. Elmaati, L. E. L. Bahir, and K. Faitah, "fault tolerant control of internal faults in wind turbine: case study of gearbox efficiency decrease," International Journal of Rotating Machinery, vol. 2018, Article ID 9538489, 8 pages, 2018.

[31] F.-D. Li, M. Wu, Y. He, and X. Chen, "Optimal control in microgrid using multi-agent reinforcement learning," ISA Transactions, vol. 51, no. 6, pp. 743-751, 2012.

[32] E. Sales-Setién and I. Peñarrocha-Alós, "Multiobjective performance-based designs in fault estimation and isolation for discrete-time systems and its application to wind turbines," International Journal of Systems Science, vol. 50, no. 6, pp. 1252-1274, 2019.

[33] E. Sales-Setien and I. Peñarrocha, "Robust estimation and diagnosis of wind turbine pitch misalignments at a wind farm 
level," Renewable Energy, vol. 146, no. 2020, pp. 1746-1765, 2019.

[34] D. Dolz, I. Penarrocha, and R. Sanchis, "Performance tradeoffs for networked jump observer-based fault diagnosis," IEEE Transactions on Signal Processing, vol. 63, no. 10, pp. 2692-2703, 2015.

[35] X. Fu, L. He, and H. Qiu, "MEMS gyroscope sensors for wind turbine blade tip deflection measurement," in Proceedings of the Instrumentation and measurement Technology Conference (I2MTC), May 2013.

[36] T. Lindstrim, M. Lomholt, J. Ostergaard et al., "Online estimation of wind turbine blade deflection with UWB signals," in Proceedings of the 2015 23rd European Signal Processing Conference (EUSIPCO), August-September, 2015.

[37] R. Poore and T. Lettenmaier, Alternative Design Study Report: WindPACT Advanced Wind Turbine Drive Train: Designs Study, Global Energy Concepts, LLC, Kirkland, WA, USA, 2002.

[38] D. Perfiliev, J. Hämäläinen, and J. Backman, "Robust analyzing tools for wind turbine blades coupled with multiobjective optimization," Journal of Energy and Power Engineering, vol. 7, pp. 1831-1836, 2013.

[39] V. Cirtoaje, A. S. Baiesu, and S. F. Mihalache, "Two controller design procedures using closed-loop Pole placement technique," Control Engineering and Applied Informatics, vol. 11, no. 1, pp. 34-43, 2009.

[40] H. Wang and S. Daley, "Actuator fault diagnosis: an adaptive observer-based technique," IEEE Transactions on Automatic Control, vol. 41, no. 7, pp. 1073-1078, 1996.

[41] J. Bernussou and G. Garcia, "Pole assignment for uncertain systems in a specified disk by output feedback," Mathematics of Control, Signals and Systems, vol. 9, no. 2, pp. 152-161, 1996.

[42] Yalmip: 2015, http://users.isy.liu.se/johanl/yalmip/.

[43] D. A. Griffin, Blade System Design Studies Volume II: Preliminary Blade Designs and Recommended Test Matrix, Global Energy Concepts, Kirkland, WA, USA, 2004.

[44] S. Simani, S. Farsoni, and P. Castaldi, "Identification for wind turbine benchmark fault diagnosis," Machines, vol. 2, no. 4, pp. 275-298, 2014.

[45] A. B. Borcehrsen, J. A. Larsen, and J. Stoustrup, "Fault detection and load distribution for the wind farm challenge," IFAC Proceedings Volumes, vol. 47, no. 3, pp. 4316-4321, 2014.

[46] J. Blesa, P. Jimenez, D. Rotondo, F. Nejjari, and V. Puig, “An interval NLPV parity equations approach for fault detection and isolation of a wind farm," IEEE Transactions on Industrial Electronics, vol. 62, no. 6, pp. 3794-3805, 2015.

[47] L. S. Duviella and M. Sayed-Mouchaweh, "An evolving classification approach for fault diagnosis and prognosis of a wind farm," in Proceedings of the 2nd Conference on Control and Fault-Tolerant Systems (SysTol), pp. 377-382, Nice, France, October 2013.

[48] D. Rotondo, F. Nejjari, and V. Puig, "A virtual actuator and sensor approach for fault tolerant control of LPV systems," Journal of Process Control, vol. 24, no. 3, pp. 203-222, 2014. 